\title{
Determining virus-host interactions and glycerol metabolism profiles in geographically diverse solar salterns with metagenomics
}

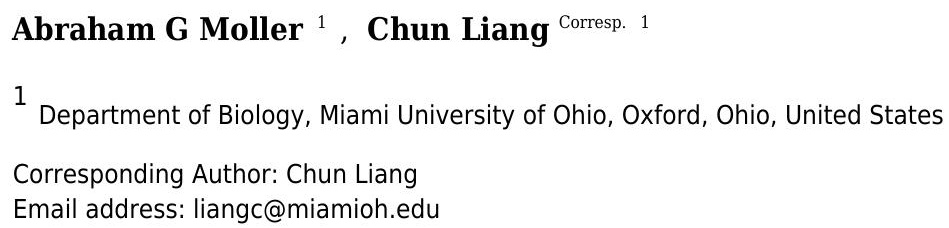

Solar salterns are excellent model ecosystems for studying virus-microbial interactions because of their low microbial diversity, environmental stability, and high viral density. By using the power of CRISPR spacers to link viruses to their prokaryotic hosts, we explored virus-host interactions in geographically diverse salterns. Using taxonomic profiling, we identified hosts such as archaeal Haloquadratum, Halorubrum, and Haloarcula and bacterial Salinibacter, and we found that community composition related to not only salinity but also local environmental dynamics. Characterizing glycerol metabolism genes in these metagenomes suggested Halorubrum and Haloquadratum possess most dihydroxyacetone kinase genes while Salinibacter possesses most glycerol-3-phosphate dehydrogenase genes. Using two different methods, we detected fewer CRISPR spacers in Haloquadratum-dominated compared with Halobacteriaceae-dominated saltern metagenomes. After CRISPR detection, spacers were aligned against haloviral genomes to map virus to host. While most alignments for each saltern metagenome linked viruses to Haloquadratum walsbyi, there were also alignments indicating interactions with the low abundance taxa Haloarcula and Haloferax. Further examination of the dinucleotide and trinucleotide usage differences between paired viruses and their hosts confirmed viruses and hosts had similar nucleotide usage signatures. Detection of cas genes in the salterns supported the possibility of CRISPR activity. Taken together, our studies suggest similar virus-host interactions exist in different solar salterns and that the glycerol metabolism gene dihydroxyacetone kinase is associated with Haloquadratum and Halorubrum. 
1 Classification: Ecology, Genomics, Bioinformatics, Computational Biology, Microbiology

2 Title: Determining virus-host interactions and glycerol metabolism profiles in

3 geographically diverse solar salterns with metagenomics

4 Authors: Abraham G Moller ${ }^{1, \dagger}$ and Chun Liang ${ }^{1, *}$

5 Author Affiliation: ${ }^{1}$ Department of Biology, Miami University, Oxford, Ohio 45056

$6 \quad{ }^{*}$ Corresponding Author: Chun Liang, liangc@miamioh.edu

7 tCurrent Address: Department of Microbiology and Immunology, Emory University,

8 Atlanta, Georgia 30322

9

10

11

12

13

14

15 


\section{Abstract}

Solar salterns are excellent model ecosystems for studying virus-microbial interactions because of their low microbial diversity, environmental stability, and high viral density. By using the power of CRISPR spacers to link viruses to their prokaryotic hosts, we explored virus-host interactions in geographically diverse salterns. Using taxonomic profiling, we identified hosts such as archaeal Haloquadratum, Halorubrum, and Haloarcula and bacterial Salinibacter, and we found that community composition related to not only salinity but also local environmental dynamics. Characterizing glycerol metabolism genes in these metagenomes suggested Halorubrum and Haloquadratum possess most dihydroxyacetone kinase genes while Salinibacter possesses most glycerol-3-phosphate dehydrogenase genes. Using two different methods, we detected fewer CRISPR spacers in Haloquadratum-dominated compared with Halobacteriaceaedominated saltern metagenomes. After CRISPR detection, spacers were aligned against haloviral genomes to map virus to host. While most alignments for each saltern metagenome linked viruses to Haloquadratum walsbyi, there were also alignments indicating interactions with the low abundance taxa Haloarcula and Haloferax. Further examination of the dinucleotide and trinucleotide usage differences between paired viruses and their hosts confirmed viruses and hosts had similar nucleotide usage signatures. Detection of cas genes in the salterns supported the possibility of CRISPR activity. Taken together, our studies suggest similar virus-host interactions exist in different solar salterns and that the glycerol metabolism gene dihydroxyacetone kinase is associated with Haloquadratum and Halorubrum. 
47

48

49

50

51

52

53

54

55

\section{Introduction}

Viruses, the most abundant entities on the planet, are critical for maintaining global biogeochemical cycles (Suttle, 2007; Rohwer, Prangishvili \& Lindell, 2009). Many viruses infect and lyse microbial hosts, releasing stored nutrients back into the ecosystem through a mechanism called the viral shunt (Suttle, 2007; Weitz \& Wilhelm, 2012). This relationship suggests viral-microbial interactions are important for environmental nutrient cycling. Clustered regularly interspaced short palindromic repeat (CRISPR) arrays, which are found in many archaeal and bacterial genomes (Grissa, Vergnaud \& Pourcel, 2007), can help us better understand the virus-host interactions that mediate nutrient cycling. Acting as an adaptive immune system against viral infection, CRISPRs incorporate short spacers cleaved from viral DNA into host genomes, providing a record of past infections and thus associations between viruses and prokaryotic hosts (Sorek, Kunin \& Hugenholtz, 2008).

Virus-host interactions are particularly important in solar saltern ecosystems. These salt-collecting ponds are hypersaline ecosystems that vary from 10 up to $37 \%$ (saturation) salt concentration by weight (Ventosa et al., 2014). The salterns at Santa Pola near Alicante, Spain contain a low diversity community of halophile microbial hosts, making it a good model ecosystem (Ventosa et al., 2014). The square archaeon Haloquadratum walsbyi and bacterioidete Salinibacter ruber are the two major components of saltern microbial communities at the highest salinities (Ventosa et al., 2015). In addition, hypersaline environments are estimated to have the highest densities of viruses $(10 \% / \mathrm{mL})$ among studied aquatic environments (Santos et al., 2012). Reduced viral decomposition in hypersaline conditions and a lack of protozoan predation may 
explain this extraordinarily high density (Santos et al., 2012). Though heterotrophic protozoans have been identified in Korean salterns (Park \& Simpson, 2015), recent studies suggest these species primarily graze upon algae, not bacteria (Heidelberg et al., 2013). Although protozoa probably do not engulf bacteria, viruses may indeed prey upon halophilic bacteria and archaea.

While saltern microbial eukaryotes may not be predators of archaea and bacteria, they do serve as the primary producers in these ecosystems. The halotolerant, unicellular green algae Dunaliella salina synthesizes vast quantities of glycerol as an osmoprotectant in response to hyperosmotic shock (Chitlaru \& Pick, 1991). Glycerol that leaks out of these algae is the primary carbon source for the bacteria and archaea that inhabit these ecosystems (Wegmann, Ben-Amotz \& Avron, 1980; Oren, 1993; Bardavid, Khristo \& Oren, 2006). The rare saltern archaeon Haloferax volcanii converts glycerol to glycerol3-phosphate and then dihydroxyacetone phosphate (DHAP) using glycerol kinase and glycerol-3-phosphate dehydrogenase, respectively (Sherwood, Cano \& Maupin-Furlow, 2009; Rawls, Martin \& Maupin-Furlow, 2011). The bacterium Salinibacter ruber, on the other hand, oxidizes glycerol to dihydroxyacetone (Sher et al., 2004; Bardavid \& Oren, 2007). Both Haloferax and Haloquadratum walsbyi can convert dihydroxyacetone to glycolysis intermediate DHAP using dihydroxyacetone kinase (Bolhuis et al., 2006; Bardavid \& Oren, 2007; Ouellette, Makkay \& Papke, 2013). Considering this metabolic context, haloviruses that lyse halophiles may promote carbon cycling as a viral shunt either by turning over glycerol metabolism intermediates or reducing rates of glycerol uptake within the ecosystem. 
In recent years, metagenomic sequencing has made it possible to study microbial

93

94

95

96

97

98 community DNA from solar salterns across the planet. Microbial DNA from the Santa Pola salterns has recently been sampled and sequenced (Fernandez et al., 2013), in addition to microbial DNA from salterns in Isla Cristina, Spain (Fernández et al., 2014b); Cahuil, Chile (Plominsky et al., 2014); and Chula Vista, California (Rodriguez-Brito et al., 2010). Metagenomics has also made it possible to study the vast haloviral populations that inhabit these ecosystems. A number of viral genomes from the CR30 (다ystallizer, $\underline{\mathbf{3 0}} \%$ salt) saltern in Santa Pola have recently been isolated and sequenced using fosmid cloning (Santos et al., 2012). Many of these fosmid clones failed to hybridize with microbial DNA, suggesting most of isolated CR30 viruses are lytic (Santos et al., 2010). Haloviral genomes have also been assembled from viral metagenomes sequenced from Lake Tyrell in Australia (Emerson et al., 2013) and the Chula Vista salterns in California (Rodriguez-Brito et al., 2010).

While metagenomic sequencing has provided extraordinary glimpses into the viral and microbial diversity of hypersaline ecosystems, it has also enabled detection of microbial CRISPR arrays. Recent studies in a variety of ecosystems have exploited CRISPRs to link environmental viruses to their microbial hosts (Tyson \& Banfield, 2008; Anderson, Brazelton \& Baross, 2011; Berg Miller et al., 2012; Emerson et al., 2013; Zhang et al., 2013; Sanguino et al., 2015; Tschitschko et al., 2015). Among these, one study examined virus-archaea interactions in an ecosystem similar to salterns - the hypersaline Lake Tyrrell, Australia (Emerson et al., 2013). Although this study showed that CRISPRs in the lake were remarkably dynamic over time, it also indicated a number of viruses could be mapped to saltern microbes with CRISPR spacers (Emerson et al., 2013). In the 
115 saltern context, the abundant community member Haloquadratum walsbyi encodes two

116 clusters of cas genes (Bolhuis et al., 2006; Dyall-Smith et al., 2011) and contains CRISPR

117 spacers that map to many Santa Pola haloviral genomes (Garcia-Heredia et al., 2012).

118 These studies suggest CRISPRs have great potential to indicate virus-archaea

119 interactions within saltern ecosystems.

120 In this study, we analyze saltern metagenomes to link haloviruses to their hosts

121 and improve our understanding of carbon cycling amongst the prokaryotes that live in

122 these environments. Furthermore, we incorporate knowledge gained from studying not

123 one but many hypersaline ecosystems across the planet. We not only use metagenomic

124 CRISPR sequences to map virus-host interactions across different saltern microbial

125 populations, but also use relevant taxonomic and functional profiling tools to determine

126 relative taxonomic abundances and relationships between taxa and glycerol metabolism

127 functions. In this context, we find common sets of virus-host interactions and glycerol

128 metabolism gene associations with particular taxa across these geographically distinct

129 salterns. We can also begin to relate lytic infection to specific glycerol metabolism

130 pathways amongst saltern microbes, thus shedding light on the viral contribution to

131 glycerol turnover and thus carbon cycling in solar salterns.

\section{Materials and Methods}

133 Obtaining metagenomic data sets and haloviral genomes. The Santa Pola, Isla

134 Cristina, and Cahuil microbial metagenomes were downloaded from the NCBI Sequence

135 Read Archive (SRA). Technical details about these microbial metagenomes are provided

136 in Table 1. Further details about sampling are described in associated publications (Ghai

137 et al., 2011; Fernandez et al., 2013; Fernández et al., 2014b,c; Plominsky et al., 2014). 
138 Chula Vista microbial metagenomes were obtained from the iMicrobe Collaborative

139 (Hurwitz, 2014) and have been previously examined in a larger study of several aquatic

140 biomes (Rodriguez-Brito et al., 2010). Chula Vista metagenomes were grouped by

141 corresponding salt concentration (low, medium, or high) and concatenated to form single

142 low (CV6-8), medium (CV12-14), and high (CV27-30) salt concentration metagenomes,

143 as shown in Table 2. The ways combined microbial metagenomes were constructed and 144 utilized in later analyses are outlined in Table 3. Santa Pola and Isla Cristina 145 metagenomes (SS13, SS19, IC21, SS33, and SS37) were analyzed individually except 146 for assembly and de novo CRISPR detection steps. The Cahuil (C34) metagenome was 147 analyzed individually throughout. All analyses performed on the microbial metagenomes 148 are outlined in Figure S1.

149 Haloviral genomes as well the Lake Tyrell viral contigs were downloaded from 150 NCBI GenBank (Table S1). Additionally, Chula Vista saltern viral metagenomes were 151 obtained through iMicrobe (Hurwitz, 2014) and then combined into one viral metagenome

152 (Table 3). This combined viral metagenome (Table 3) was then assembled with Newbler 153 version 2.9 into Chula Vista viral contigs that were also included in the haloviral library 154 (Table S1).

155 Profiling taxonomic and functional compositions of selected metagenomes. The 156 selected individual metagenomes (Tables 1 and 2) were profiled for relative taxonomic 157 abundances with MetaPhyler (Liu et al., 2011a). MetaPhyler aligns reads against a library 158 of clade-specific marker genes. Reads that align against a marker gene above a particular 159 threshold alignment score are then binned to the corresponding taxon (e.g., 160 Haloquadratum, for reads aligning to Haloquadratum marker genes). Taxonomic profiles 
161 were further compared in MATLAB using principal component analysis (PCA). Clusters

162 identified with PCA were evaluated for statistical significance using the ADONIS R

163 function from the $R$ package vegan (Oksanen et al., 2016) loaded in $R$ version 3.2.2 ( $R$

164 Core Team, 2015). The significance of salinity in structuring the similarities between

165 sample taxonomic profiles was determined using the envfit and ordisurf functions from

166 the R package vegan (Oksanen et al., 2016), which fit vectors and smooth surfaces onto

167 an ordination, respectively. Envfit was used with default parameters, while the number of

168 knots (degrees of freedom plus one) for ordisurf was set to nine due to the nine

169 metagenomes examined. The percent of reads mapping to markers for each

170 metagenome were also calculated and compared to detect differences in the proportions

171 of reads used for taxonomic profiling depending on metagenome.

172 The metagenomes were further profiled for functional abundances with ShotMAP

173 (Nayfach et al., 2015) and metAnnotate (Petrenko et al., 2015). ShotMAP determination

174 of gene family abundance was conducted with a Hidden Markov Model (HMM)-based

175 search algorithm against an indexed library of Pfam family HMMs. Abundances were

176 calculated from the numbers of metagenomic reads mapping to particular Pfam families

177 normalized by the length of each Pfam target gene in the gene family. We selected

178 abundances of nine gene families known to be related to glycerol metabolism for further

179 analysis - dihydroxyacetone kinase (Dak1 - PF02733; Dak1_2 - PF13684; Dak2 -

180 PF02734), NAD+-dependent glycerol dehydrogenase (Fe-ADH - PF00465; Fe-ADH_2 -

181 PF13685), NAD+-dependent glycerol-3-phosphate dehydrogenase (both $\mathrm{N}$ - and $\mathrm{C}$ -

182 terminal domains: NAD_Gly3p_dh_N - PF01210; NAD_Gly3p_dh_C - PF07479), and

183 glycerol kinase (FGGY_N - PF00370; FGGY_C - PF02782), listed both with Pfam family 
184 names and ID numbers. Relative abundances were then analyzed for correlations with 185 respect to salinity using Microsoft Excel. metAnnotate (Petrenko et al., 2015) was used to determine the abundance of particular Pfam gene families for particular taxa. Like ShotMAP, metAnnotate uses an HMM-based search algorithm to find reads that match certain Pfam gene family HMMs. Unlike ShotMAP, however, it also determines the taxonomic affiliations of these gene family reads, and thus determines which taxa have which gene families. We used metAnnotate to taxonomically profile nine aforementioned gene families known to be related to glycerol metabolism. We then calculated the proportion of reads mapping to each taxon among all those mapping to a gene family to determine the taxonomic profiles of these gene families. These gene family profiles were then visualized as heatmaps and hierarchically clustered with the $\mathrm{R}$ pheatmap package (Kolde, 2015). Clusters were evaluated for statistical significance with the ADONIS R function (Oksanen et al., 2016).

De novo and reference-guided CRISPR detection. CRISPR arrays were detected using two different methods (reference-guided and de novo). All CRISPR detection analyses were treated as qualitative comparisons amongst sites because of limited sample size and inability to fully correct for differences in sequencing depth amongst metagenomes. Because CRISPR arrays' repetitive nature makes them highly difficult to assemble, CRISPR arrays were detected purely from unassembled metagenomic reads. De novo detection was performed with Crass (Skennerton, Imelfort \& Tyson, 2013) with default parameters, which identifies repeat-spacer-repeat patterns in individual reads, uses these as "seeds" to recruit other CRISPR-containing reads, and then assembles these reads into CRISPR arrays. We further clustered Crass spacers using CD-HIT (Li \& 
207 Godzik, 2006, p.) with a similarity threshold of 0.9 and aligned them against known direct

208

209

210

211

212

213

214

215

216

217

218

219

220

221

222

223

224

225

226

227

228

229

repeats from CRISPRdb (Grissa, Vergnaud \& Pourcel, 2007) to determine the associated host organism.

Because Crass does not directly indicate the putative host encoding the detected CRISPRs, we developed a pipeline (MetaCRAST) to constrain spacer detection by expected host species (Moller \& Liang, 2016). MetaCRAST searched each read for direct repeat (DR) sequences matching query DRs specified by the user. These query DRs were selected from known CRISPR DRs. In general, the query DR sequences might be known to be present in CRISPR arrays found in genomes expected from the taxonomic profile; for example, known genomic Haloquadratum walsbyi DRs might be used to search a metagenome for Haloquadratum walsbyi CRISPRs. Direct repeats within a certain Levenshtein edit distance (i.e., number of insertions, deletions, or substitutions necessary to convert one sequence to another) were identified in reads using the WuManber multi-pattern search algorithm (Wu, Manber \& Myers, 1995).

In the first step of the pipeline, reads that contain a single DR sequence matching the user-specified DR were detected with the Wu-Manber algorithm and collected for further analysis. In the second step of the pipeline, individual reads gathered in the first step were searched for two or more copies of the query direct repeat sequence within appropriate distances from each other. In the case of CRISPRs, direct repeats have been found to be between 23 and 47 bp long, while spacers, and thus the distances between DRs, have been found to be between 26 and 50 bp long (Skennerton, Imelfort \& Tyson, 2013). In the third step, the sequence fragments between the direct repeats were extracted as the spacers. These spacers were then collected as a non-redundant set in 
230 a hash, and then further clustered into non-redundant sets using CD-HIT (Li \& Godzik, 231 2006).

MetaCRAST was implemented in Perl and takes a set of query direct repeats and

233 a metagenome (each in FASTA format) as file inputs. It is publically available for 234 download at GitHub (https://github.com/molleraj/MetaCRAST), where all command line 235 parameters are also documented. It has also been validated against simulated 236 metagenomes and tested against two real metagenomes corresponding to acid mine 237 drainage (AMD) and enhanced biological phosphorus removal (EBPR) ecosystems 238 (Moller \& Liang, 2016).

We performed reference-guided CRISPR detection with MetaCRAST using the 240 following parameters. We used a group of 29 DR sequences previously detected in 241 Haloferacales and Halobacteriales genomes (Table S4) in the genomic CRISPR 242 database CRISPRdb (Grissa, Vergnaud \& Pourcel, 2007) as query DRs. We thus 243 searched for reads containing sequences matching any of these 29 direct repeat 244 sequences. The maximum allowed distance between detected direct repeats (i.e., 245 maximum spacer length) was set to $60 \mathrm{bp}$. Acceptable edit distance - the allowed 246 differences (insertions, deletions, or substitutions) between query and detected read DR 247 sequences - were adjusted from 0 to 3 to see how variation in edit distance affected 248 reference-guided CRISPR detection. All spacers detected for each condition (for each 249 metagenome and acceptable error number) were then clustered using CD-HIT with a 250 clustering similarity threshold of 0.9 , which effectively clusters spacers together into a 251 non-redundant set based on a pairwise similarity of $90 \%$ or greater. 
252 Mapping virus-host interactions with CRISPR spacers. CRISPR spacers extracted

253 from the metagenomes with Crass and reference-guided methods and clustered with CD-

254 HIT were then aligned against the library of haloviral genomes using NCBI BLAST

255 (Altschul et al., 1990) with an e-value cutoff of 1e-03. All spacers detected with Crass for

256 the combined Santa Pola dataset (see Table 3) were aligned against this library to create

257 its corresponding interaction network. For several individual metagenomes (e.g., SS13,

258 SS19), no Crass spacers mapped to the haloviral genomes, making the more sensitive

259 reference-guided search (MetaCRAST) necessary. Thus, for individual metagenomes,

260 spacers detected with MetaCRAST using a maximum Levenshtein edit distance of 3 were

261 aligned for network creation. BLAST results from each method were then processed to

262 form lists of virus-host pairs. Networks of virus-host interactions were drawn with

263 Cytoscape (Shannon et al., 2003).

264 Comparative analysis of virus and host sequence characteristics. Viruses and hosts

265 matched through CRISPR mapping were further compared in nucleotide k-mer

266 frequencies to determine whether virus and host frequencies were similar. Dinucleotide

267 and trinucleotide usages were determined using the function nmercount from the

268 MATLAB Bioinformatics Toolbox (MATLAB and Bioinformatics Toolbox, Release 2015a).

269 Dinucleotide and trinucleotide frequencies were compared for a non-redundant list of

270 viruses associated to each of the six detected hosts listed below. All viruses were

271 associated to possible hosts using our in-house reference-based CRISPR detection

272 method. Haloquadratum walsbyi C23, Haloferax volcanii DS2, Halogeometricum

273 borinquense DSM 11551, Halorhabdus utahensis DSM 12940, Haloarcula marismortui

274 ATCC 43049 (chromosome 1), and Haloarcula hispanica N601 (chromosome 1) host 
275 genomes were analyzed along with the matched haloviral genomes. To measure the

276 sequence characteristic differences among virus-host pairs, each virus dinucleotide and

277 trinucleotide frequency was subtracted from each corresponding host genome frequency

278 and squared. All these squared distances were summed for possible dinucleotides and

279 for possible trinucleotides to generate an overall Euclidean measure of distance between

280 virus and host.

281 Assembling metagenomes and detecting halobacterial cas genes in metagenomic

282 contigs. Combined Santa Pola, combined Chula Vista, and Cahuil metagenomes (Table

2833 outlines combined metagenomes) were assembled into contigs to detect cas operons.

284 Combined Santa Pola, combined Chula Vista, and Cahuil metagenomes were assembled

285 both with Newbler and Velvet, creating a total of six sets of contigs (Zerbino \& Birney,

286 2008). Both Newbler and Velvet were used to compare the effects of Overlap-Layout-

287 Consensus (Newbler) and de Bruijn graph (Velvet) assembly methods. Newbler assembly

288 was performed with default parameters, while Velvet assembly was performed with a k-

289 mer size of 31.

290 Newbler and Velvet contigs assembled from each combined metagenome were

291 first aligned against cas genes annotated in Haloferax volcanii DS2, Halorubrum

292 lacusprofundi ATCC 49239, and Haloquadratum walsbyi C23 genomes using NCBI blastn

293 (Altschul et al., 1990) with an e-value cutoff of 1e-06 and NCBI tblastx with an e-value

294 cutoff of 1e-30. Color-coded cas detection results for genes in the Haloquadratum,

295 Halorubrum, and Haloferax cas operons were drawn with the synthetic biology tool

296 Pigeon (Bhatia \& Densmore, 2013). A library of cas genes from the three major

297 halobacterial orders (the Haloferacales, Halobacteriales, and Natrialbales) were 
298 downloaded from NCBI Genbank (see Tables S13-S15) and aligned against each set of

299 assembled contigs (assembled with each method and from each combined metagenome)

300 to determine the taxonomic affiliation of matching cas genes. BLAST alignments were

301 performed with the same parameters described above. Taxonomy information was

302 extracted from matching cas gene Genbank records with the NCBI Efetch utility to

303 determine the taxonomic affiliations of the assembled cas contigs.

304 Results and Discussion

305 Taxonomic profiling identifies predominant members of saltern microbial

306 communities. We profiled the taxonomic abundances of metagenomes sequenced from

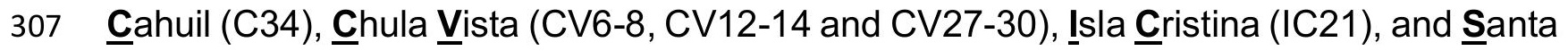

308 Pola Saltern (SS13, SS19, SS33, SS37) sites (where the ID numbers indicate salinity

309 percentages) to better understand the population structures of saltern microbial

310 communities and to compare host abundances amongst sites. We found the low salinity

311 Chula Vista (CV6-8) and SS13 salterns had different microbial communities from the

312 other salterns, containing large proportions of bacterial Actinobacteria, Proteobacteria

313 and Rhodobacteraceae $(7.64,14.28$, and $20.69 \%$ in SS13 and $6.6,10.05$, and $11.13 \%$ in

314 CV6-8, respectively - see Figure 1A). In addition, we found Haloquadratum was the major

315 taxon identified in the SS19, SS33, and SS37 salterns (88.32, 65.55, and $78.06 \%$

316 abundance, respectively), while Halorubrum and Halobacteriaceae were major

317 components of the Cahuil and IC21 salterns (31.79 and $64.55 \%$ in Cahuil; 32.5 and

$31851.63 \%$ in IC21, respectively - see Figure 1A). Bacterial Salinibacter was identified in the

319 SS19, SS33, and SS37 salterns $(2.18,7.16$, and $6.82 \%$ relative abundances,

320 respectively). While Haloquadratum was the major identified taxon in the higher salinity 
321 Santa Pola salterns, Halobacteriaceae composed the most abundant identified taxon

322 identified in the medium and high salinity Chula Vista salterns (51.46 and $77.02 \%$,

323 respectively). We realize that the Halobacteriaceae component of the saltern

324 communities may also include species in the new order Haloferacales (Gupta, Naushad

325 \& Baker, 2015), but this taxon was defined after the MetaPhyler marker library was

326 constructed (Liu et al., 2011a). These taxonomic profiles and clustering results further

327 guided our analyses of host CRISPR arrays, as we used taxonomy to guide our searches

328 for CRISPR spacers.

329 We further compared the taxonomic profiles through principal component analysis,

330 which found that the first two principal components accounted for $88 \%$ of variation

331 amongst the examined taxonomic profiles. PCA grouped the SS19, SS33, and SS37

332 profiles; low salinity Chula Vista (CV6-8) and SS13 profiles; and medium salinity Chula

333 Vista (CV12-14), high salinity Chula Vista (CV27-30), IC21, and C34 profiles (Figure 1B).

334 These groups were found to be statistically significant in their between-group differences

335 (ADONIS; $F=19.817 ; p=0.002$ ). We furthermore found percent salinity to be a significant

336 feature in structuring the similarities amongst metagenomes when fitting this variable to

337 the PCA ordination either as a vector or a response surface (envfit: $p=0.038$; ordisurf,

338 knots $=9: p=0.0291)$.

339 We also determined the percentage of reads mapping to phylogenetic markers to

340 detect read length-dependent differences in taxonomic profiling among metagenomes

341 (Figure S2). The percentage mapping was similar among Santa Pola, Isla Cristina, and

342 Cahuil (C34) metagenomes (an average of $0.51 \%$ of reads with a standard deviation of $343 \quad 0.0483 \%$ among these metagenomes), but lower among the Chula Vista metagenomes 
344 (an average of $0.21 \%$ of reads with a standard deviation of $0.041 \%$ among these

345 metagenomes) due to lower coverage (see Tables 1 and 2).

We have thus built upon prior metagenomics studies to show that Haloquadratum

347 is the majority taxon in many salterns (Oh et al., 2009), particularly those that are

348 environmentally stable with high salinity (Figure 1). The Santa Pola and Isla Cristina

349 metagenomes have previously been taxonomically profiled through mapping of reads to

350 16S rRNAs obtained from the Ribosomal Database Project (Fernandez et al., 2013;

351 Fernández et al., 2014b; Ventosa et al., 2014). These 16S rRNA profiling studies revealed

352 that the lowest salinity saltern (SS13) contained a community of bacterial Actinobacteria

353 and Proteobacteria and archaeal Euryarchaeota, while members of the Euryarchaeota

354 increased in abundance with increasing salinity (Fernández et al., 2014c,a; Ventosa et

355 al., 2015). Compared with the Santa Pola salterns, Cahuil and Isla Cristina salterns may

356 be more dynamic, with more rapidly changing daily and yearly temperatures and higher

357 rainfall (Fernández et al., 2014c). This may lead to lower concentrations of potassium,

358 magnesium, and sulfate in those salterns, thus leading to lower abundances of

359 Haloquadratum, and higher abundances of other haloarchaea (Podell et al., 2014;

360 Fernández et al., 2014c).

361 Functional profiling reveals abundances of glycerol metabolism genes and their

362 taxonomic affiliations. Glycerol is the main carbon source for saltern microbes (Oren,

363 1993). We used two functional profiling tools to study the glycerol metabolism genes

364 critical for carbon cycling in these ecosystems. We used the tool ShotMAP to determine

365 abundances of glycerol metabolism gene families (Nayfach et al., 2015) and metAnnotate

366 to study the taxonomic distribution of reads mapping to these gene families (Petrenko et 
367 al., 2015). As with MetaPhyler, Cahuil, Isla Cristina, and Santa Pola metagenomic reads

368 mapped to the ShotMAP reference library in higher proportions than the Chula Vista reads

369 (Figure S3). Using these tools, we examined the abundances and taxonomic affiliations

370 of the glycerol kinase (FGGY_N and FGGY_C), NAD+-dependent glycerol-3-phosphate

371 dehydrogenase (NAD_Gly3P_dh_N and NAD_Gly3P_dh_C), glycerol dehydrogenase

372 (iron-containing alcohol dehydrogenase - Fe-ADH and Fe-ADH_2), and

373 dihydroxyacetone kinase (Dak1, Dak1_2, and Dak2) Pfam gene families.

We first determined gene family abundances with ShotMAP and then examined

relationships between these abundances and salinity levels. Among the gene families we

examined, we noticed both negative and positive correlations between relative gene family abundance and salinity (Figure 2). Dak1, Dak2, FGGY_N, and Fe-ADH_2 families showed positive correlations with salinity, while Dak1_2, NAD_Gly3P_dh_N, NAD_Gly3P_dh_C, FGGY_C, and Fe-ADH families showed negative correlations with salinity. Only Dak1_2,NAD_Gly3P_dh_N, and NAD_Gly3P_dh_C gene families showed significant correlations with salinity $(r=-0.617,-0.795$, and $-0.787 ; p=0.038,0.005$, and 0.006, respectively). We also noted family abundances calculated for the Chula Vista metagenomes to be larger than those found for any other metagenome. More short reads mapping to these gene families may account for these higher family abundances than those found for the Santa Pola, Isla Cristina, and Cahuil metagenomes.

We next determined the taxonomic affiliations of reads mapping to these gene families using metAnnotate. We generated heat maps of these affiliations hierarchically clustered both with regard to metagenome (Figure 3A) and gene family (Figure 3B). As with taxonomic profiles, the taxonomic affiliations of glycerol metabolism genes in the 
390

391

392

393

394

395

396

397

398

399

400

401

402

403

404

405

406

407

408

409

410

411

Haloquadratum-rich SS19, SS33, and SS37 metagenomes group together, as do those of the Chula Vista metagenomes, and the SS13, IC21, and Cahuil (C34) metagenomes (Figure 3A). Across gene family, taxonomic affiliations of the dihydroxyacetone kinase families were closest to each other, as well as those of the alcohol dehydrogenase (Fe$\mathrm{ADH}$ ) and glycerol kinase (FGGY) gene families (Figure 3B). The two $\mathrm{NAD}^{+}$-dependent glycerol-3-phosphate dehydrogenase families grouped together, but many reads mapping to these families were found to be unclassified (Figure 3B). These described gene family and metagenome clusters were found to be statistically significant (ADONIS; $\mathrm{F}=7.5468$ and 2.8768; $\mathrm{p}=0.014$ and 0.013 , for gene family and metagenome clusters, respectively).

We also analyzed these taxonomic affiliations for each gene family across each individual metagenome (Figure 3C). Haloquadratum associated with dihydroxyacetone kinase gene families in the SS19, SS33, and SS37 metagenomes while Halorubrum associated with these families in the IC21 and Cahuil metagenomes (Figure 3C). The two $\mathrm{NAD}^{+}$-dependent glycerol-3-phosphate dehydrogenase families, on the other hand, associated mainly to Salinibacter in the Cahuil (C34), high salinity Chula Vista, SS19, SS33, and SS37 metagenomes and to Spiribacter in the SS13 and IC21 metagenomes. Unlike the previous families, alcohol dehydrogenase and glycerol kinase had less clear associations across the metagenomes, with association to Haloquadratum and Halorubrum but also many unclassified reads. In addition, glycerol kinase families associated to Spiribacter (SS13 and IC21) and the alcohol dehydrogenase family to Halobellus (C34, CV27-30, SS19, SS33, and SS37) across several metagenomes. 
Our functional studies thus found both positive and negative correlations between

413 glycerol metabolism gene family abundances and salinity (Figure 2). Prior studies of the

414 Santa Pola and Isla Cristina salterns found that glycerol metabolism genes, such as 415 dihydroxyacetone kinase, glycerol kinase, and NAD+-dependent glycerol-3-phosphate dehydrogenase, increased in abundance with salt concentrations (Fernández et al., 417 2014c,a). Taxonomic affiliations, however, differed amongst salterns and different gene 418 families (Figure 3). Dihydroxyacetone kinase most strongly associated with Haloquadratum, except in the IC21 and Cahuil (C34) salterns, where it associated 420 strongly with Halorubrum instead. NAD+-dependent glycerol-3-phosphate dehydrogenase, on the other hand, associated with Salinibacter (Figure 3C). Based on prior understanding of saltern glycerol cycling (Oren, 1993, 1994; Sher et al., 2004), these results together suggest archaea such as Haloquadratum and Halorubrum consume the glycerol metabolism intermediate dihydroxyacetone in these ecosystems, while bacteria such as Salinibacter may first phosphorylate and then oxidize glycerol to metabolize it 426 (Sher et al., 2004).

Metagenomic CRISPRs detected with two independent methods show variation in in metagenomes using both de novo and reference-guided methods. We used Crass for 430 de novo detection (Skennerton, Imelfort \& Tyson, 2013) and a custom pipeline (MetaCRAST) for reference-guided detection (see Materials and Methods). We used a combination of Halobacteriales and Haloferacales CRISPR direct repeats downloaded from CRISPRdb as our query for MetaCRAST analyses across all metagenomes. 
Overall, more spacers were detected in IC21 and Cahuil (C34) metagenomes

435

436

437

438

439

440

441

442

443

444

445

446

447

448

449

450

451

452

453

454

455

456

compared with the high salinity Chula Vista, SS13, SS19, SS33, and SS37 metagenomes

(Figure 4) both with MetaCRAST ( 3 errors allowed) and Crass. More spacers were detected with Crass compared to reference-guided methods in the combined Santa Pola, SS13, and SS33 metagenomes (377 vs. 277,29 vs. 9 , and 146 vs. 80 spacers, respectively). For the reference-guided detection analyses, the number of spacers identified increased as the allowable error (insertions, deletions, or substitutions different from the query DR sequence) in alignment was increased from 0 to 3 (Figure 4). No spacers were detected with either method in the low or medium salinity Chula Vista metagenomes. When we normalized by total number of metagenomic reads, we found that the Cahuil (C34) metagenome had the largest number of spacers detected per million reads (Figure S4). Nonetheless, it is important to note that CRISPR detection analyses could only be treated as qualitative rather than quantitative because of limited sample size and inability to fully correct for differences in sequencing depth amongst metagenomes.

We then compared the detected direct repeats and spacers among metagenomes to discover common CRISPR array types or spacers (Figures 5 and 6). Remarkably, common spacers were identified amongst the SS19 and SS37 metagenomes (13 with Crass and 18 with MetaCRAST among these two, respectively) using both detection methods (Figures $5 \mathrm{~A}$ and $5 \mathrm{~B}$ ). Amongst the metagenomes with a higher number of detected spacers (IC21 and Cahuil/C34), common spacers were detected with both methods (5 in IC21 and 24 in Cahuil - see Figures 5C and 5D). Amongst the direct repeats detected with Crass in the combined Chula Vista, combined Santa Pola, and 
457 Cahuil metagenomes, there was one direct repeat (DR) sequence common to both Cahuil

458 and combined Santa Pola (Figure 6). We also searched the combined Santa Pola direct

459 repeats against a full database of direct repeats from CRISPRdb to determine possible 460 taxonomic affiliations. We found that 14 of the 67 detected combined Santa Pola DRs 461 matched DRs in the CRISPRdb database (Table S3). Of these 14 matching DRs, 10 462 matched haloarchaeal DRs, while 4 matched bacterial DRs.

463 Our CRISPR detection results showed a smaller number of CRISPR spacers found 464 in salterns where Haloquadratum was identified as the majority taxon compared with 465 salterns where Halobacteriaceae was found to be the majority taxon. Furthermore, 466 comparison of detected metagenomic spacers to those found in the genomes of two 467 different $H$. walsbyi strains (C23 and DSM 16790) suggests variation in CRISPR array 468 length in the salterns. Strain C23 contains a full set of cas genes and several CRISPR 469 arrays, while strain DSM 16790 lost all its cas genes and all but five spacers (Dyall-Smith 470 et al., 2011). While we could not identify any C23 spacers amongst our metagenomes, 471 we could find at least three DSM 16790 spacers in several metagenomes. A successful 472 mobile element integration event in spite of CRISPR defense is thought to have promoted 473 loss of spacers in Haloquadratum genomes (Dyall-Smith et al., 2011). Taken together, 474 this evidence suggests genomic CRISPR arrays in Haloquadratum species may vary in 475 length.

476 Metagenomic CRISPRs detected with two independent methods map haloviruses 477 to saltern hosts.

478 We then aligned the spacers detected with both methods against a library of 479 haloviral genomes (Table S1) to determine virus-host interactions in these salterns. 
480 Unlike CRISPR spacers detected with Crass in several individual Santa Pola 481 metagenomes, spacers detected in a combination of all Santa Pola and Isla Cristina 482 metagenomes did align to haloviral genomes (Figure 7). In this Crass analysis of the 483 combined Santa Pola dataset, we detected virus interactions most likely with 484 Haloquadratum (G467), Haloarcula (G42 and G82), and Saccharomonospora (G57) 485 hosts, based on our previous comparison of Crass DRs (listed by group number, such as 486 G467) against CRISPRdb DRs (Figure 7). For many of the interactions (e.g., G467, G42), 487 we could determine a possible host by such comparison. For several notable groups of 488 interactions, however, we could not determine possible hosts (e.g., G85, G1290).

While Crass only provided a picture of virus-host interactions in the combined 490 Santa Pola metagenome, MetaCRAST detected spacers that mapped to haloviral 491 genomes in each of the individual Santa Pola, Isla Cristina, and Cahuil metagenomes (Figure 8). Only one interaction was detected in the SS13 dataset (Figure 8A), but there 493 were strikingly similar groups of virus-host interactions detected in the SS19, SS33, and SS37 metagenomes (Figures 8B, 8C, and 8D). While many groups of interactions were 495 similar to those expected from the Haloquadratum walsbyi strain C23 CRISPR array (Figure S5), no C23 spacers matched any detected metagenomic spacers, while at least three Haloquadratum walsbyi strain DSM 16790 spacers did match spacers detected in SS19, IC21, SS33, and SS37 metagenomes. Aside from Haloquadratum, in the SS33 metagenome, we also detected Haloarcula-virus interactions with eHP-4, eHP-8, and 500 eHP-13 (Figure 8C). Quite surprisingly, we noticed remarkably different virus501 Halobacteriaceae interactions in the IC21 and Cahuil (C34) metagenomes (Figures 8E 502 and 8F), perhaps due to differences in taxonomic composition at the genus level. 
Our CRISPR mapping results not only showed consistent viral interactions with

504

505

506

507

508

509

510

511

512

513

514

515

516

517

518

519

520

521

522

523

524

525

Haloquadratum across salterns but also numerous interactions with less abundant taxa such as Haloferax and Haloarcula. As expected from our comparison of taxonomic and functional profiles, there were consistent sets of interactions amongst SS19, SS33, and SS37 salterns, where Haloquadratum species form the majority of the microbial population (Figure 8). These interactions suggest similar viral infection patterns across these environments, consistent with "global pond" theories of microbial and viral exchange (Dyall-Smith et al., 2011; Atanasova et al., 2012). Unclear biological requirements for CRISPR spacer incorporation make it difficult to make any conclusion about the abundances of the viruses. Though some models suggest viruses must have reached a critical number within their hosts to induce CRISPR spacer incorporation into host genomes (Garrett, Vestergaard \& Shah, 2011), characterization of the Haloferax volcanii CRISPR systems suggests plasmid copy number has little effect on incorporation (Maier et al., 2013).

There were also interactions detectable with Crass that could not map to any known hosts (Figure 7). Some of these hosts may be poorly characterized members of the Nanohaloarchaea, as recent studies have suggested for eHP-12 and eHP-16 (Martínez-García et al., 2014). Surprisingly, we did not detect any spacers or virus-host interactions with Halorubrum with either CRISPR detection method. Either limitations of our detection tools or a lack of Halorubrum CRISPRs could account for this observation. Nucleotide usage comparison suggests biological plausibility of virus-host associations. To determine whether our virus-host mappings are biologically plausible, we compared the dinucleotide and trinucleotide frequencies of CRISPR-determined virus- 
526 host pairings. Over evolutionary time, viruses' nucleotide usage steadily approaches that

527 of the host, reflecting adaptation to host codon usage, tRNA availability, intracellular

528 nucleotide pools, and host restriction modification systems (Edwards et al., 2015). We

529 compared these nucleotide usage frequencies both among the predicted hosts and

530 analyzed metagenomes (Figure 9). Among predicted hosts, Haloquadratum walsbyi,

531 Haloferax volcanii, and Haloarcula marismortui showed the smallest overall differences

532 in dimer and trimer usage, suggesting strong virus-host association, while Haloarcula

533 hispanica and Halorhabdus utahensis showed the highest overall differences (Figures 9A

534 and 9B). Only one virus associated to Halogeometricum borinquense, and it had a greater

535 difference in trimer frequency compared with dimer frequency relative to the other

536 predicted hosts. Among the metagenomes, the smallest differences were found in the

537 SS33 metagenome; the next smallest differences were found in the Cahuil (C34), SS19,

538 and SS37 metagenomes, while the largest difference was found in the single virus-host

539 pair detected in the SS13 metagenome (Figures 9C and 9D).

540 Assembled metagenomic contigs contain halobacterial cas genes. To further

541 confirm our CRISPR detection and mapping results, we searched through metagenomic

542 contigs for the cas genes required for CRISPR activity. Because all microbial

543 metagenomes studied were sequenced with 454 technology, and Newbler is designed to

544 assemble 454 reads, we expected longer contigs to be assembled with Newbler and

545 found this in our assembly results (Table S2). We both searched for contigs that matched

546 several different haloarchaeal cas operons (Figure 10A) and profiled the taxonomic

547 affiliations of haloarchaeal cas genes that best matched contigs (Figure 10B). We were

548 able to assemble almost all genes in cas operons in three different metagenomes (Cahuil, 
549 combined Chula Vista, and combined Santa Pola) from several different Type I-B and

550 Type I-D cas operons (Figure 10A).

551 We also searched a library of cas genes from three different haloarchaeal orders

552 (Halobacteriales, Haloferacales, and Natrialbales) and generated profiles of taxonomic

553 affiliations for the genes that best matched contigs (Figure 10B). Haloarcula composed a

554 large proportion of Halobacteriales contig affiliations, especially amongst the Cahuil (C34)

555 contigs ( $71.4 \%$ among Newbler contigs; $41.4 \%$ among Velvet contigs). On the other hand,

556 Haloquadratum and Halorubrum composed large proportions of the Haloferacales

557 annotations, especially for the combined Santa Pola dataset (14.5\% and $25.8 \%$ for

558 Newbler contigs; $23.5 \%$ and $26.6 \%$ for Velvet contigs). We also calculated the total

559 number of hits in the search against the cas gene libraries (Figure S6) and profiled the

560 taxonomic affiliations of all cas gene alignments to the contigs (Figure S7). There were

561 smaller Haloarcula, Haloferax, Halorubrum, and Haloquadratum proportions in the total

562 hit profiles (Figure S7) compared with the best hit profiles (Figure 10B).

563 Conclusions

564 Taken together, our results suggest common sets of virus-host interactions across

565 different salterns and variation in CRISPR antiviral defenses amongst saltern microbes.

566 While common sets of viruses infect haloarchaea across salterns, these hosts may have

567 strikingly different levels of CRISPR defenses. Haloquadratum, it seems, may exist in

568 either virus-resistant or virus-sensitive strains depending on the presence or absence of

569 its CRISPR/Cas system. CRISPR/Cas systems may be part of the previously examined

570 Haloquadratum accessory gene pool (Legault et al., 2006). While it is surprising that virus-

571 sensitive strains would be present in such a virus-rich environment, even these forms 
572 may have some innate halovirus resistance through extensive genomic variation of phage

573 recognition proteins (Cuadros-Orellana et al., 2007). These patterns may not apply to

574 other haloarchaea and saltern inhabitants, however. While Haloquadratum encodes

575 CRISPRs chromosomally, Haloarcula and Haloferax encode CRISPR elements on both

576 their chromosomes and plasmids, suggesting the possibility of horizontally transferred

577 CRISPR systems (Baliga et al., 2004; Grissa, Vergnaud \& Pourcel, 2007; Hartman et al.,

578 2010; Liu et al., 2011b; Han et al., 2012). Viral interactions with Salinibacter remain far

579 more elusive, as this bacterium lacks a CRISPR system (Grissa, Vergnaud \& Pourcel, 580 2007).

Our CRISPR virus-host interaction studies help explain the paradox of high viral

582 density and low infection rates in hypersaline ecosystems (Brum et al., 2005; Bettarel et

al., 2011). Some studies have suggested high rates of lysogeny accounts for this paradox

(Bettarel et al., 2011), while others suggest saltern microbes have increased levels of viral resistance (Brum et al., 2005). Santa Pola saltern viruses have not been shown to hybridize to saltern microbes' genomes, excluding the lysogeny hypothesis (Santos et al.,

587 2010). Our results suggest CRISPR defenses may have a role in reducing infection rates, as detected CRISPR spacers were mapped to known, sequenced saltern phages and 589 CRISPR-associated (cas) genes were detected in studied saltern metagenomes, 590 indicating saltern microbes do have CRISPR systems. Nonetheless, we still cannot exclude the possibility that innate phage resistance due to variation in cell surface proteins could also account for low viral infection rates (Cuadros-Orellana et al., 2007). Nonetheless, the variation of CRISPR systems and resulting variation in 594 Haloquadratum phage resistance could carefully control the archaeon's abundance. 
595 Together both haloviruses and CRISPR defense could tightly regulate the population of 596 Haloquadratum, which is both the major component of many salterns and consumer of

597 the glycerol metabolism intermediate dihydroxyacetone. Levels of CRISPR defense may 598 thus account for the turnover of this organism. In combination with our studies of glycerol 599 metabolism, our insights into virus-host interactions may also show how lytic viruses 600 regulate glycerol turnover in these ecosystems. Our glycerol metabolism gene detection 601 results suggest Haloquadratum and Halorubrum tend to oxidize and then phosphorylate 602 glycerol, while other Halobacteriaceae and Salinibacter first phosphorylate and then 603 oxidize glycerol. Knowing that previously identified Santa Pola haloviruses are lytic 604 (Santos et al., 2010), viruses we mapped to Haloquadratum could either reduce overall 605 rates of glycerol oxidation to dihydroxyacetone or release free dihydroxyacetone back 606 into the ecosystem through lytic infection.

607 Future studies should further analyze the number and genomic arrangement of 608 spacers in different Haloquadratum strains and examine how abundances of these 609 populations change over time. Ideally, these studies would also relate changes in 610 CRISPR arrays to changes in viral population structure over time. Such work would build upon recent studies of the temporal dynamics of virus-host interactions in Lake Tyrrell, an Australian hypersaline ecosystem (Emerson et al., 2013). Our studies and such future 613 projects will help us better understand how viruses regulate hypersaline ecosystems 614 across the planet.

\section{Acknowledgments}

616 Thanks to Michael Crowder and Gary Lorigan (Miami University) for feedback on the 617 project and manuscript. 


\section{References}

619 Altschul SF., Gish W., Miller W., Myers EW., Lipman DJ. 1990. Basic local alignment

620 search tool. Journal of Molecular Biology 215:403-410. DOI: 10.1016/S0022-

621 2836(05)80360-2.

622

623

624

625

626

627

628

629

630

631

632

633

634

635

636

637

638

639

Anderson RE., Brazelton WJ., Baross JA. 2011. Using CRISPRs as a metagenomic tool to identify microbial hosts of a diffuse flow hydrothermal vent viral assemblage: CRISPR spacers reveal hosts of marine vent viral assemblage. FEMS Microbiology Ecology 77:120-133. DOI: 10.1111/j.1574-6941.2011.01090.x.

Atanasova NS., Roine E., Oren A., Bamford DH., Oksanen HM. 2012. Global network of specific virus-host interactions in hypersaline environments: Haloviruses are promiscuous. Environmental Microbiology 14:426-440. DOI: 10.1111/j.14622920.2011.02603.x.

Baliga NS., Bonneau R., Facciotti MT., Pan M., Glusman G., Deutsch EW., Shannon P., Chiu Y., Weng RS., Gan RR., Hung P., Date SV., Marcotte E., Hood L., Ng WV. 2004. Genome sequence of Haloarcula marismortui: A halophilic archaeon from the Dead Sea. Genome Research 14:2221-2234. DOI: 10.1101/gr.2700304.

Bardavid RE., Khristo P., Oren A. 2006. Interrelationships between Dunaliella and halophilic prokaryotes in saltern crystallizer ponds. Extremophiles 12:5-14. DOI: 10.1007/s00792-006-0053-y.

Bardavid RE., Oren A. 2007. Dihydroxyacetone metabolism in Salinibacter ruber and in Haloquadratum walsbyi. Extremophiles 12:125-131. DOI: 10.1007/s00792-0070114-x. 
640 Berg Miller ME., Yeoman CJ., Chia N., Tringe SG., Angly FE., Edwards RA., Flint HJ.,

641

642

643

644

645

646

647

648

649

650

651

652

653

654

655

656

657

658

659

660

661

662

Lamed R., Bayer EA., White BA. 2012. Phage-bacteria relationships and CRISPR elements revealed by a metagenomic survey of the rumen microbiome: Rumen viral metagenome. Environmental Microbiology 14:207-227. DOI: 10.1111/j.1462-2920.2011.02593.x.

Bettarel Y., Bouvier T., Bouvier C., Carré C., Desnues A., Domaizon I., Jacquet S., Robin A., Sime-Ngando T. 2011. Ecological traits of planktonic viruses and prokaryotes along a full-salinity gradient. FEMS Microbiology Ecology 76:360372. DOI: 10.1111/j.1574-6941.2011.01054.x.

Bhatia S., Densmore D. 2013. Pigeon: A Design Visualizer for Synthetic Biology. ACS Synthetic Biology 2:348-350. DOI: 10.1021/sb400024s.

Bolhuis H., Palm P., Wende A., Falb M., Rampp M., Rodriguez-Valera F., Pfeiffer F., Oesterhelt D. 2006. The genome of the square archaeon Haloquadratum walsbyi : life at the limits of water activity. BMC Genomics 7:169. DOI: 10.1186/1471-2164-7-169.

Brum JR., Steward GF., Jiang SC., Jellison R. 2005. Spatial and temporal variability of prokaryotes, viruses, and viral infections of prokaryotes in an alkaline, hypersaline lake. Aquatic Microbial Ecology 41:247-260. DOI: 10.3354/ame041247.

Chitlaru E., Pick U. 1991. Regulation of Glycerol Synthesis in Response to Osmotic Changes in Dunaliella. Plant Physiology 96:50-60. DOI: 10.1104/pp.96.1.50. Cuadros-Orellana S., Martin-Cuadrado A-B., Legault B., D’Auria G., Zhaxybayeva O., Papke RT., Rodriguez-Valera F. 2007. Genomic plasticity in prokaryotes: the 
663

664

665

666

667

668

669

670

671

672

673

674

675

676

677

678

679

680

681

682

683

684

685

case of the square haloarchaeon. The ISME Journal 1:235-245. DOI:

10.1038/ismej.2007.35.

Dyall-Smith ML., Pfeiffer F., Klee K., Palm P., Gross K., Schuster SC., Rampp M., Oesterhelt D. 2011. Haloquadratum walsbyi : Limited Diversity in a Global Pond. PLOS ONE 6:e20968. DOI: 10.1371/journal.pone.0020968.

Edwards RA., McNair K., Faust K., Raes J., Dutilh BE. 2015. Computational approaches to predict bacteriophage-host relationships. FEMS Microbiology Reviews:fuv048. DOI: 10.1093/femsre/fuv048.

Emerson JB., Andrade K., Thomas BC., Norman A., Allen EE., Heidelberg KB., Banfield JF. 2013. Virus-Host and CRISPR Dynamics in Archaea-Dominated Hypersaline Lake Tyrrell, Victoria, Australia. Archaea 2013:1-12. DOI: 10.1155/2013/370871.

Fernandez AB., Ghai R., Martin-Cuadrado AB., Sanchez-Porro C., Rodriguez-Valera F., Ventosa A. 2013. Metagenome Sequencing of Prokaryotic Microbiota from Two Hypersaline Ponds of a Marine Saltern in Santa Pola, Spain. Genome Announcements 1:e00933-13. DOI: 10.1128/genomeA.00933-13.

Fernández AB., Ghai R., Martin-Cuadrado A-B., Sánchez-Porro C., Rodriguez-Valera F., Ventosa A. 2014a. Prokaryotic taxonomic and metabolic diversity of an intermediate salinity hypersaline habitat assessed by metagenomics. FEMS Microbiology Ecology 88:623-635. DOI: 10.1111/1574-6941.12329.

Fernández AB., León MJ., Vera B., Sánchez-Porro C., Ventosa A. 2014b. Metagenomic Sequence of Prokaryotic Microbiota from an Intermediate-Salinity Pond of a Saltern in Isla Cristina, Spain. Genome Announcements 2:e00045-14. DOI: 10.1128/genomeA.00045-14. 
686 Fernández AB., Vera-Gargallo B., Sánchez-Porro C., Ghai R., Papke RT., Rodriguez-

687 Valera F., Ventosa A. 2014c. Comparison of prokaryotic community structure

688 from Mediterranean and Atlantic saltern concentrator ponds by a metagenomic

689 approach. Frontiers in Microbiology 5. DOI: 10.3389/fmicb.2014.00196.

Garcia-Heredia I., Martin-Cuadrado A-B., Mojica FJM., Santos F., Mira A., Antón J., 691

692

693

694

695

696

697

698

699

700

701

702

703

704

705

706

707

Grissa I., Vergnaud G., Pourcel C. 2007. The CRISPRdb database and tools to display CRISPRs and to generate dictionaries of spacers and repeats. BMC Bioinformatics 8:172. DOI: 10.1186/1471-2105-8-172.

Gupta RS., Naushad S., Baker S. 2015. Phylogenomic analyses and molecular signatures for the class Halobacteria and its two major clades: a proposal for division of the class Halobacteria into an emended order Halobacteriales and two new orders, Haloferacales ord. nov. and Natrialbales ord. nov., containing the 
novel families Haloferacaceae fam. nov. and Natrialbaceae fam. nov. INTERNATIONAL JOURNAL OF SYSTEMATIC AND EVOLUTIONARY MICROBIOLOGY 65:1050-1069. DOI: 10.1099/ijs.0.070136-0.

Han J., Zhang F., Hou J., Liu X., Li M., Liu H., Cai L., Zhang B., Chen Y., Zhou J., Hu S., Xiang H. 2012. Complete Genome Sequence of the Metabolically Versatile Halophilic Archaeon Haloferax mediterranei, a Poly(3-Hydroxybutyrate-co-3Hydroxyvalerate) Producer. Journal of Bacteriology 194:4463-4464. DOI: 10.1128/JB.00880-12.

Hartman AL., Norais C., Badger JH., Delmas S., Haldenby S., Madupu R., Robinson J., Khouri H., Ren Q., Lowe TM., Maupin-Furlow J., Pohlschroder M., Daniels C., Pfeiffer F., Allers T., Eisen JA. 2010. The Complete Genome Sequence of Haloferax volcanii DS2, a Model Archaeon. PLoS ONE 5:e9605. DOI: 10.1371/journal.pone.0009605.

Heidelberg KB., Nelson WC., Holm JB., Eisenkolb N., Andrade K., Emerson JB. 2013. Characterization of eukaryotic microbial diversity in hypersaline Lake Tyrrell, Australia. Frontiers in Microbiology 4. DOI: 10.3389/fmicb.2013.00115.

Hurwitz B. 2014. iMicrobe: Advancing Clinical and Environmental Microbial Research using the iPlant Cyberinfrastructure. In: Plant and Animal Genome,.

Kolde R. 2015. pheatmap: Pretty Heatmaps.

Legault BA., Lopez-Lopez A., Alba-Casado JC., Doolittle WF., Bolhuis H., RodriguezValera F., Papke RT. 2006. Environmental genomics of "Haloquadratum walsbyi" in a saltern crystallizer indicates a large pool of accessory genes in an otherwise coherent species. BMC Genomics 7:1-13. DOI: 10.1186/1471-2164-7-171. 
732 Li W., Godzik A. 2006. Cd-hit: a fast program for clustering and comparing large sets of

733 protein or nucleotide sequences. Bioinformatics 22:1658-1659. DOI:

734

735

736

737

738

739

740

741

742

743

744

745

746

747

748

749

750

751

752

753

Maier L-K., Lange SJ., Stoll B., Haas KA., Fischer SM., Fischer E., Duchardt-Ferner E., Wöhnert J., Backofen R., Marchfelder A. 2013. Essential requirements for the detection and degradation of invaders by the Haloferax volcanii CRISPR/Cas system I-B. RNA Biology 10:865-874. DOI: 10.4161/rna.24282.

Martínez-García M., Santos F., Moreno-Paz M., Parro V., Antón J. 2014. Unveiling viral-host interactions within the "microbial dark matter." Nature Communications 5:4542. DOI: $10.1038 /$ ncomms5542.

MATLAB and Bioinformatics Toolbox, Release 2015a The MathWorks, Inc.: Natick, Massachusetts, United States.

Moller AG., Liang C. 2016. MetaCRAST: Reference-guided extraction of CRISPR spacers from unassembled metagenomes. PeerJ Preprints 4:e2278v2. DOI: 10.7287/peerj.preprints.2278v2. 
754 Nayfach S., Bradley PH., Wyman SK., Laurent TJ., Williams A., Eisen JA., Pollard KS.,

755 Sharpton TJ. 2015. Automated and Accurate Estimation of Gene Family

756

757

Abundance from Shotgun Metagenomes. PLoS Comput Biol 11:e1004573. DOI:

758 10.1371/journal.pcbi.1004573.

Oh D., Porter K., Russ B., Burns D., Dyall-Smith M. 2009. Diversity of Haloquadratum

759

760

761

762

763

764

765

766

767

768

769

770

771

772

773

774

775 and other haloarchaea in three, geographically distant, Australian saltern crystallizer ponds. Extremophiles 14:161-169. DOI: 10.1007/s00792-009-0295-6.

Oksanen J., Blanchet FG., Friendly M., Kindt R., Legendre P., McGlinn D., Minchin PR., O’Hara RB., Simpson GL., Solymos P., Stevens MHH., Szoecs E., Wagner H. 2016. vegan: Community Ecology Package.

Oren A. 1993. Availability, uptake and turnover of glycerol in hypersaline environments. FEMS Microbiology Ecology 12:15-23. DOI: 10.1111/j.15746941.1993.tb00012.x.

Oren A. 1994. The ecology of the extremely halophilic archaea. FEMS Microbiology Reviews 13:415-439. DOI: 10.1111/j.1574-6976.1994.tb00060.x.

Ouellette M., Makkay AM., Papke RT. 2013. Dihydroxyacetone metabolism in Haloferax volcanii. Frontiers in Microbiology 4. DOI: 10.3389/fmicb.2013.00376.

Park JS., Simpson AGB. 2015. Diversity of Heterotrophic Protists from Extremely Hypersaline Habitats. Protist 166:422-437. DOI: 10.1016/j.protis.2015.06.001.

Petrenko P., Lobb B., Kurtz DA., Neufeld JD., Doxey AC. 2015. MetAnnotate: functionspecific taxonomic profiling and comparison of metagenomes. BMC Biology 13:92. DOI: 10.1186/s12915-015-0195-4. 
776 Plominsky AM., Delherbe N., Ugalde JA., Allen EE., Blanchet M., Ikeda P., Santibanez

777 F., Hanselmann K., Ulloa O., De la Iglesia R., von Dassow P., Astorga M.,

778

779

780

781

782

783

784

785

786

787

788

789

790

791

792

793

794

795

796

797

798

Galvez MJ., Gonzalez ML., Henriquez-Castillo C., Vaulot D., Lopes do Santos A., van den Engh G., Gimpel C., Bertoglio F., Delgado Y., Docmac F., Elizondo-

Patrone C., Narvaez S., Sorroche F., Rojas-Herrera M., Trefault N. 2014.

Metagenome Sequencing of the Microbial Community of a Solar Saltern

Crystallizer Pond at Cahuil Lagoon, Chile. Genome Announcements 2:e01172-

14-e01172-14. DOI: 10.1128/genomeA.01172-14.

Podell S., Emerson JB., Jones CM., Ugalde JA., Welch S., Heidelberg KB., Banfield JF., Allen EE. 2014. Seasonal fluctuations in ionic concentrations drive microbial succession in a hypersaline lake community. The ISME Journal 8:979-990. DOI: 10.1038/ismej.2013.221.

R Core Team 2015. R: A Language and Environment for Statistical Computing. Vienna, Austria: R Foundation for Statistical Computing.

Rawls KS., Martin JH., Maupin-Furlow JA. 2011. Activity and Transcriptional Regulation of Bacterial Protein-Like Glycerol-3-Phosphate Dehydrogenase of the Haloarchaea in Haloferax volcanii. Journal of Bacteriology 193:4469-4476. DOI: 10.1128/JB.00276-11.

Rodriguez-Brito B., Li L., Wegley L., Furlan M., Angly F., Breitbart M., Buchanan J., Desnues C., Dinsdale E., Edwards R., Felts B., Haynes M., Liu H., Lipson D., Mahaffy J., Martin-Cuadrado AB., Mira A., Nulton J., Pašić L., Rayhawk S., Rodriguez-Mueller J., Rodriguez-Valera F., Salamon P., Srinagesh S., Thingstad TF., Tran T., Thurber RV., Willner D., Youle M., Rohwer F. 2010. Viral and 
799

800

801

802

803

804

805

806

807

808

809

810

811

812

813

814

815

816

817

818

819

820

821

microbial community dynamics in four aquatic environments. The ISME Journal 4:739-751. DOI: 10.1038/ismej.2010.1.

Rohwer F., Prangishvili D., Lindell D. 2009. Roles of viruses in the environment. Environmental Microbiology 11:2771-2774. DOI: 10.1111/j.14622920.2009.02101.x.

Sanguino L., Franqueville L., Vogel TM., Larose C. 2015. Linking environmental prokaryotic viruses and their host through CRISPRs. FEMS Microbiology Ecology 91:fiv046. DOI: 10.1093/femsec/fiv046.

Santos F., Yarza P., Parro V., Briones C., Antón J. 2010. The metavirome of a hypersaline environment: The metavirome of a hypersaline environment. Environmental Microbiology 12:2965-2976. DOI: 10.1111/j.14622920.2010.02273.x.

Santos F., Yarza P., Parro V., Meseguer I., Rosselló-Móra R., Antón J. 2012. CultureIndependent Approaches for Studying Viruses from Hypersaline Environments. Applied and Environmental Microbiology 78:1635-1643. DOI: 10.1128/AEM.07175-11.

Shannon P., Markiel A., Ozier O., Baliga NS., Wang JT., Ramage D., Amin N., Schwikowski B., Ideker T. 2003. Cytoscape: A Software Environment for Integrated Models of Biomolecular Interaction Networks. Genome Research 13:2498-2504. DOI: 10.1101/gr.1239303.

Sher J., Elevi R., Mana L., Oren A. 2004. Glycerol metabolism in the extremely halophilic bacterium Salinibacter ruber. FEMS Microbiology Letters 232:211-215. DOI: 10.1016/S0378-1097(04)00077-1. 
822 Sherwood KE., Cano DJ., Maupin-Furlow JA. 2009. Glycerol-Mediated Repression of

823 Glucose Metabolism and Glycerol Kinase as the Sole Route of Glycerol

824 Catabolism in the Haloarchaeon Haloferax volcanii. Journal of Bacteriology

825 191:4307-4315. DOI: 10.1128/JB.00131-09.

826 Skennerton CT., Imelfort M., Tyson GW. 2013. Crass: identification and reconstruction

827

828

829

830

831

832

833

834

835

836

837

838

839

840

841

842

843

of CRISPR from unassembled metagenomic data. Nucleic Acids Research 41:e105-e105. DOI: 10.1093/nar/gkt183.

Sorek R., Kunin V., Hugenholtz P. 2008. CRISPR - a widespread system that provides acquired resistance against phages in bacteria and archaea. Nature Reviews Microbiology 6:181-186. DOI: 10.1038/nrmicro1793.

Suttle CA. 2007. Marine viruses - major players in the global ecosystem. Nature Reviews Microbiology 5:801-812. DOI: 10.1038/nrmicro1750.

Tschitschko B., Williams TJ., Allen MA., Páez-Espino D., Kyrpides N., Zhong L., Raftery MJ., Cavicchioli R. 2015. Antarctic archaea-virus interactions: metaproteome-led analysis of invasion, evasion and adaptation. The ISME Journal 9:2094-2107. DOI: 10.1038/ismej.2015.110.

Tyson GW., Banfield JF. 2008. Rapidly evolving CRISPRs implicated in acquired resistance of microorganisms to viruses. Environmental Microbiology 10:200207. DOI: 10.1111/j.1462-2920.2007.01444.x.

Ventosa A., Fernández AB., León MJ., Sánchez-Porro C., Rodriguez-Valera F. 2014. The Santa Pola saltern as a model for studying the microbiota of hypersaline environments. Extremophiles 18:811-824. DOI: 10.1007/s00792-014-0681-6. 
844 Ventosa A., de la Haba RR., Sánchez-Porro C., Papke RT. 2015. Microbial diversity of 845 hypersaline environments: a metagenomic approach. Current Opinion in Microbiology 25:80-87. DOI: 10.1016/j.mib.2015.05.002.

847 Wegmann K., Ben-Amotz A., Avron M. 1980. Effect of Temperature on Glycerol 848 Retention in the Halotolerant Algae Dunaliella and Asteromonas. Plant Physiology 66:1196-1197. DOI: 10.1104/pp.66.6.1196.

850

851

852

853

854

855

856

857

858

859

860

Weitz JS., Wilhelm SW. 2012. Ocean viruses and their effects on microbial communities and biogeochemical cycles. F1000 Biology Reports 4. DOI: 10.3410/B4-17.

Wu S., Manber U., Myers E. 1995. A Subquadratic Algorithm for Approximate Regular Expression Matching. Journal of Algorithms 19:346-360. DOI: 10.1006/jagm.1995.1041.

Zerbino DR., Birney E. 2008. Velvet: Algorithms for de novo short read assembly using de Bruijn graphs. Genome Research 18:821-829. DOI: 10.1101/gr.074492.107.

Zhang Q., Rho M., Tang H., Doak TG., Ye Y. 2013. CRISPR-Cas systems target a diverse collection of invasive mobile genetic elements in human microbiomes. Genome Biology 14:R40. DOI: 10.1186/gb-2013-14-4-r40. 


\section{Figure 1 (on next page)}

Taxonomic profiles (A) and principal component analysis (B) of selected saltern metagenomes.

Metagenomes were profiled using MetaPhyler and resulting profiles clustered using MATLAB. Profiles show the relative abundances (in percent) of 11 different taxa identified with MetaPhyler (A). Profiles are grouped by geographic locations - Cahuil, Chula Vista, and Santa Pola (A). The first two principal components, which together account for $88 \%$ of variation in the profiles, are shown in the PCA results (B). Three different groups of similar profiles were identified with PCA (blue - CV6-8, SS13; orange - CV12-14, IC21, C34, CV27-30; red - SS33, SS37, SS19). These groups were found to be statistically significant in their between-group differences (ADONIS; $F=19.817 ; p=0.002$ ). 


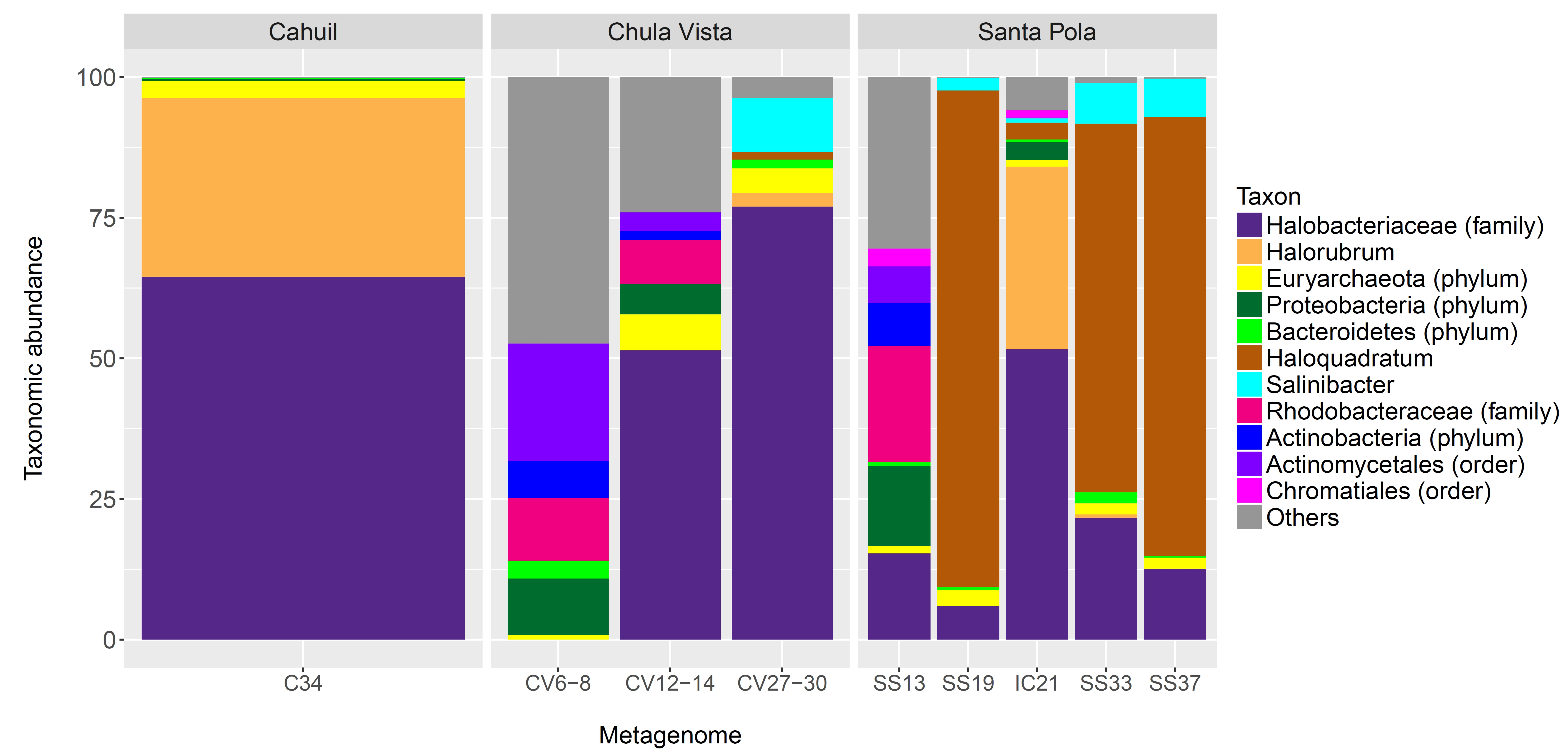

B

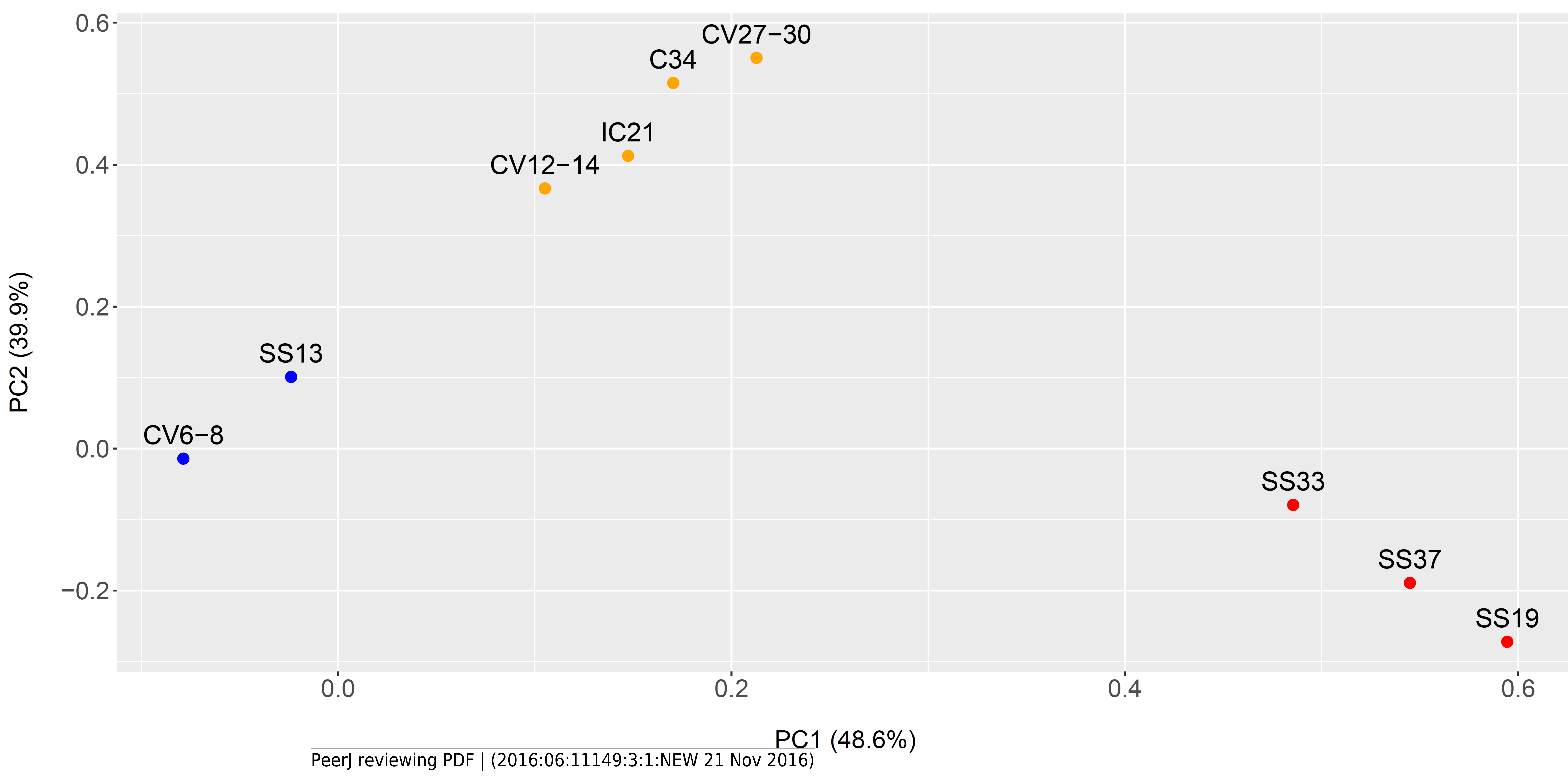




\section{Figure 2 (on next page)}

Analysis of correlations between percent salinity and relative gene family abundances determined with ShotMAP.

We determined relative abundances of nine different gene families using ShotMAP (glycerol kinase, FGGY_N and FGGY_C; NAD+-dependent glycerol-3-phosphate dehydrogenase, NAD_Gly3P_dh_N and NAD_Gly3P_dh_C; glycerol dehydrogenase/iron-containing alcohol dehydrogenase, Fe-ADH and Fe-ADH_2; and dihydroxyacetone kinase, Dak1, Dak1_2, and Dak2). Relative gene family abundance (listed on the y-axis) was calculated as the sum of hits to target genes within a gene family each normalized by target gene length. Correlations between relative gene family abundance and percent salinity (weight per volume) and respective $p$-values were determined with Microsoft Excel. 

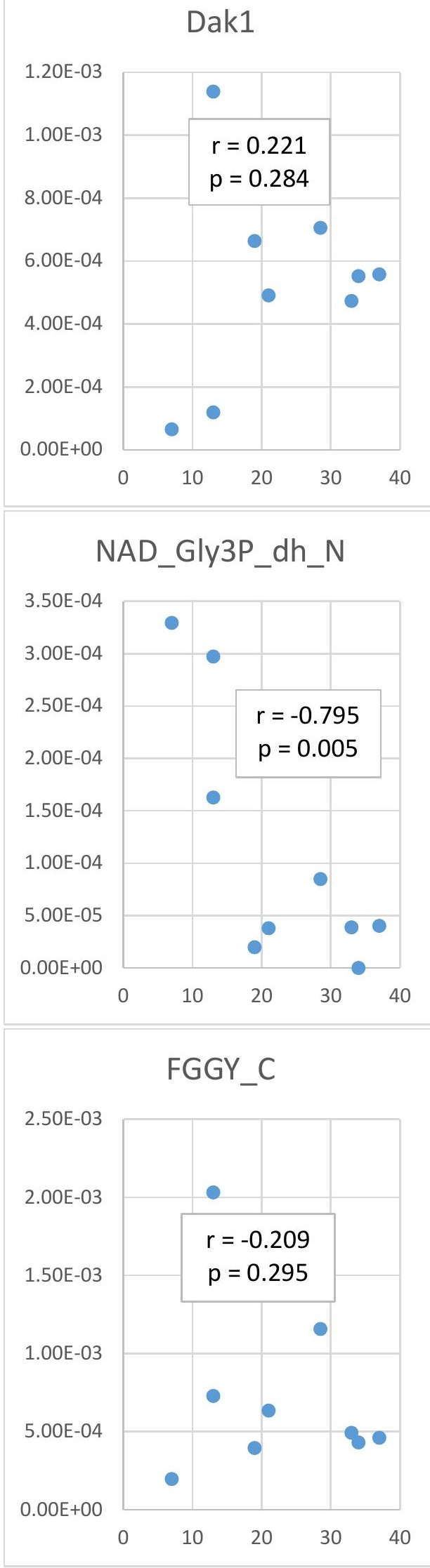
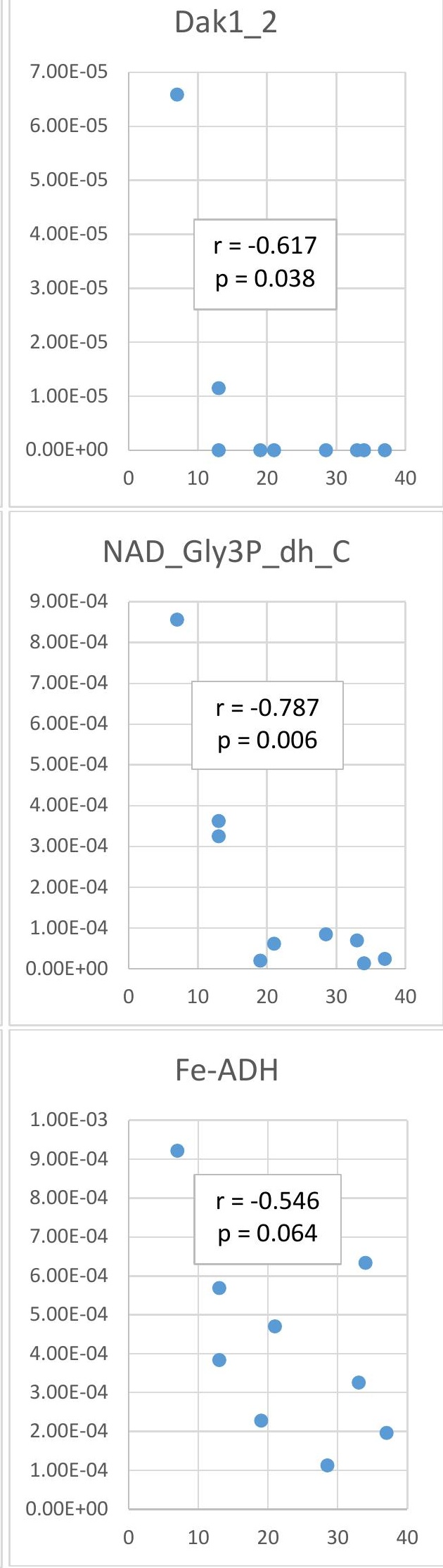

Dak2
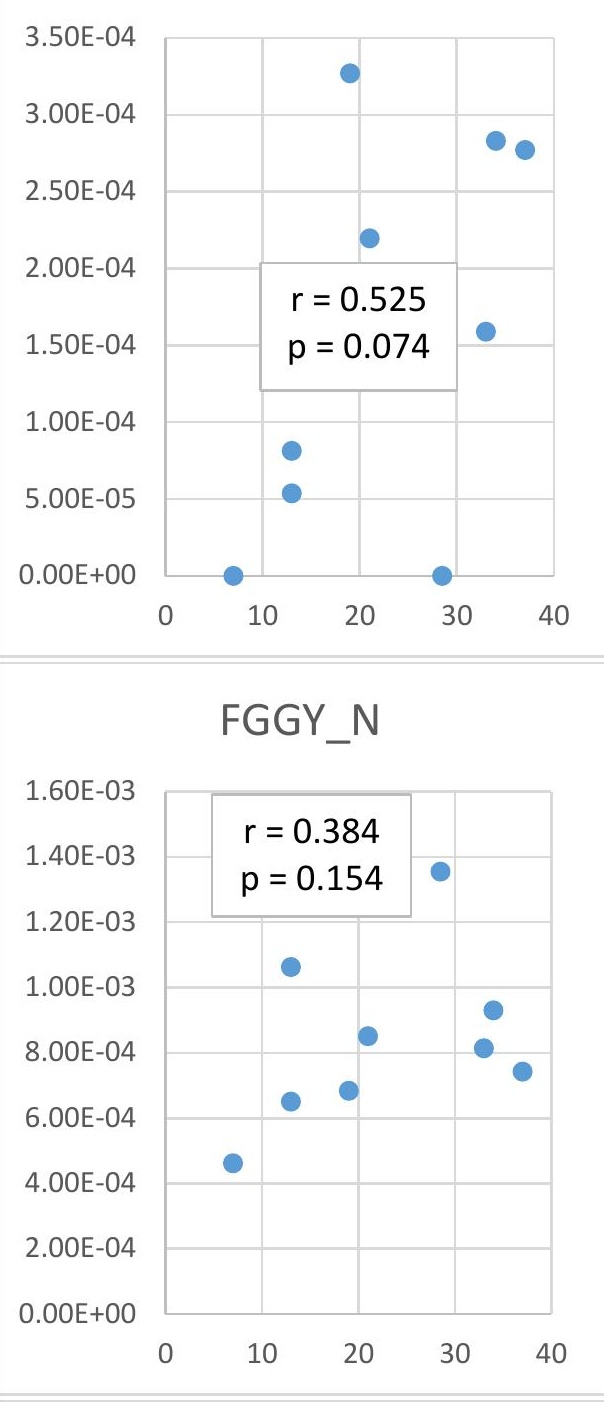

Fe-ADH_2

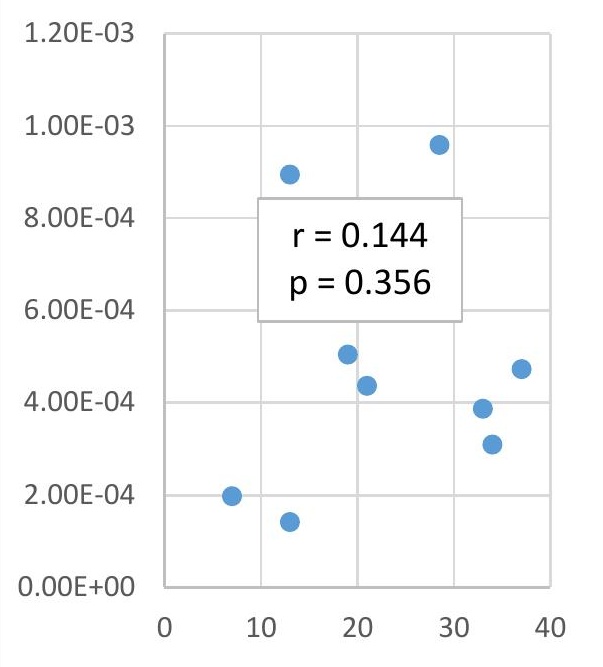

\section{Percent Salinity (w/v)}




\section{Figure 3 (on next page)}

Heat map visualizations of glycerol metabolism taxonomic profiles determined with metAnnotate.

We determined taxonomic profiles of nine different gene families using metAnnotate (glycerol kinase, FGGY_N and FGGY_C; NAD+-dependent glycerol-3-phosphate dehydrogenase, NAD_Gly3P_dh_N and NAD_Gly3P_dh_C; glycerol dehydrogenase/ironcontaining alcohol dehydrogenase, Fe-ADH and Fe-ADH_2; and dihydroxyacetone kinase, Dak1, Dak1_2, and Dak2). Relative gene family abundance (referred to as level in C) here is defined as the fraction of all hits for a gene family mapping to a particular taxon. Abundance profiles were determined for genus and site (A), genus and gene family (B), and genus, site, and gene family (C). Only site and gene family profiles (A and B) were hierarchically clustered. The primary gene family and metagenome (site) clusters (see Results) were found to be statistically significant (ADONIS; $F=7.5468$ and 2.8768; $p=0.014$ and 0.013 , for gene family and metagenome clusters, respectively). The first two heat maps ( $A$ and $B$ ) were generated with the heatmap R package, while the last heat map (C) was generated with ggplot2. 



Metagenome 
Figure 4 (on next page)

Number of CRISPR spacers detected in each metagenome after clustering with CD-HIT.

Spacers were detected either with the de novo detection method (Crass) or the referenceguided method (MetaCRAST) with corresponding maximum edit distances (from 0 to 3 ) described in Materials and Methods that used a query of 29 halobacterial CRISPR direct repeat sequences. All spacer counts are reported after clustering initially detected spacers with CD-HIT (with a clustering similarity threshold of 0.9 ). 


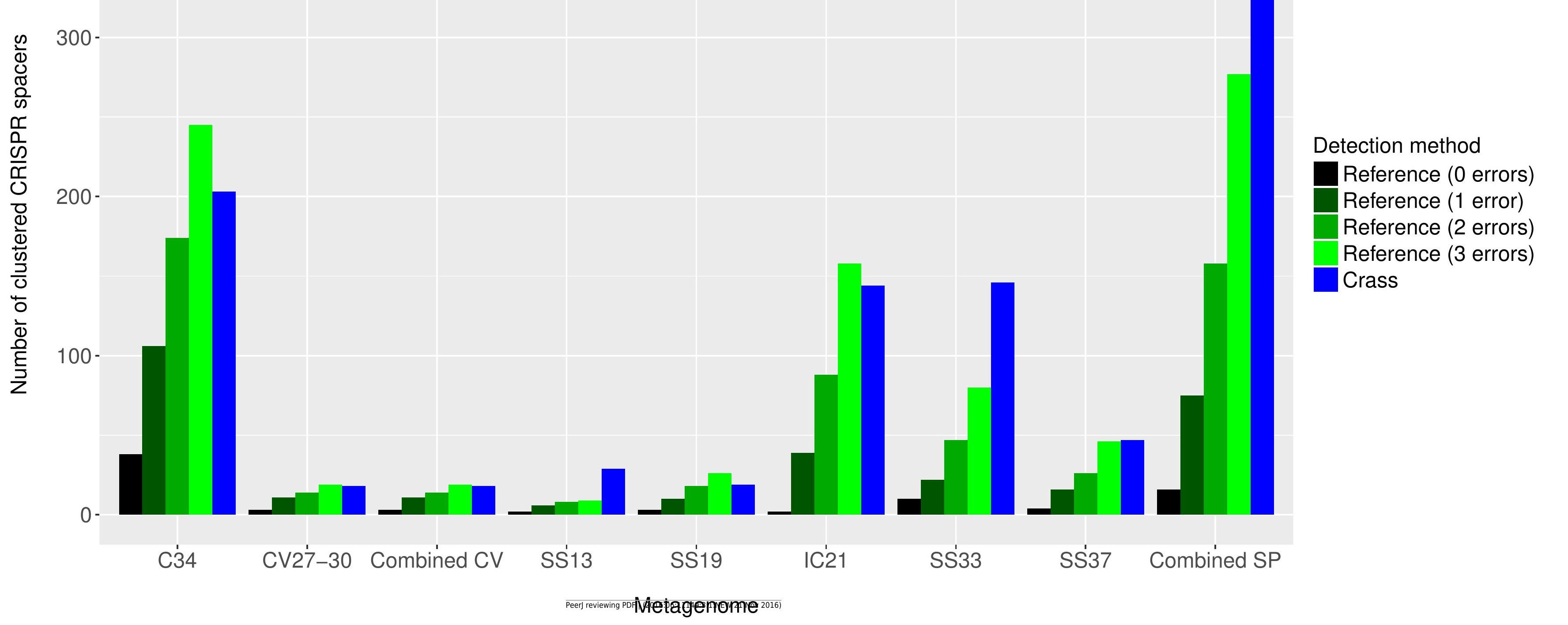


Figure $\mathbf{5}$ (on next page)

Comparison of CRISPR spacers detected with de novo (Crass) and reference-guided (MetaCRAST) methods.

All reference-guided spacers compared here with Crass spacers were detected with a maximum allowed edit distance of 3 (insertions, deletions, or mismatches with query direct repeat sequence). CRISPR spacers detected with de novo (A) and reference-guided (B) methods in SS13, SS19, SS33, and SS37 metagenomes were compared amongst sites to determine identical spacers. For the IC21 (C) and Cahuil/C34 (D) metagenomes, spacers were compared between the two detection methods. 


\section{A}

\section{B Manuscript to be reviewed}
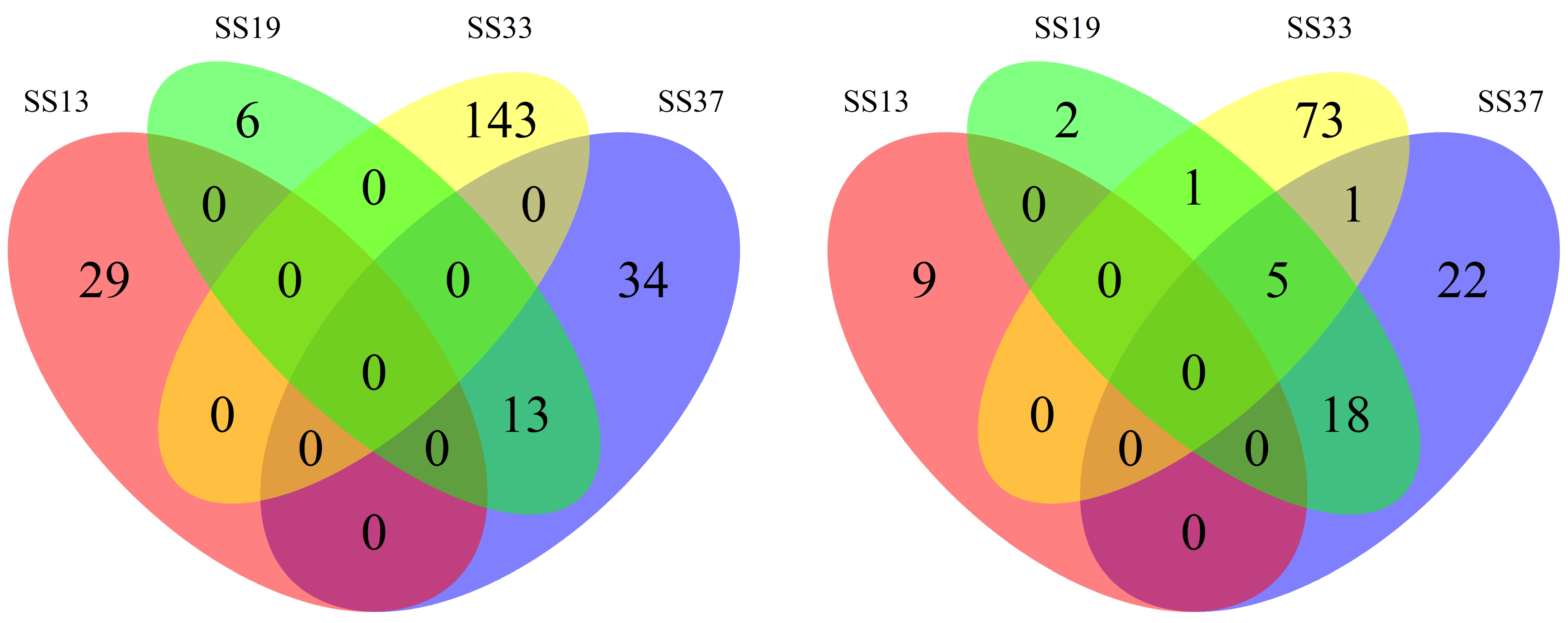

C

\section{D}
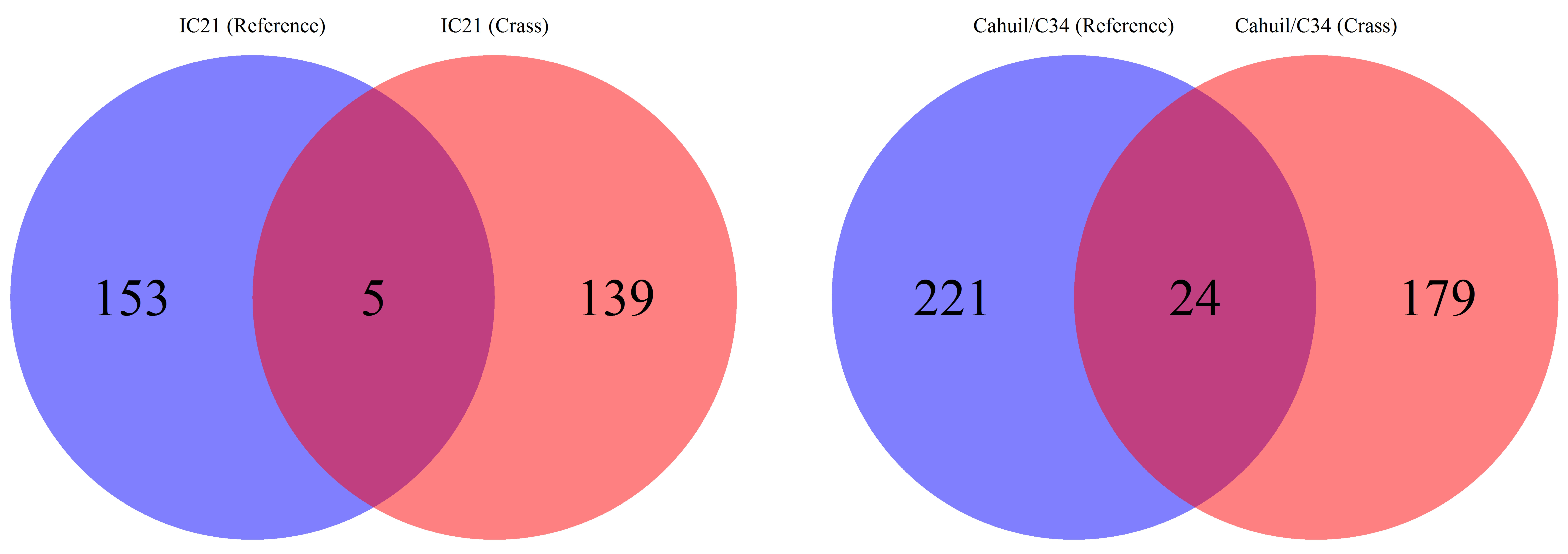


\section{Figure 6}

Comparison of CRISPR direct repeats detected with the de novo (Crass) method.

Direct repeats detected with Crass were compared amongst Cahuil (C34), combined Chula Vista, and combined Santa Pola and Isla Cristina metagenomes listed in Table 3.

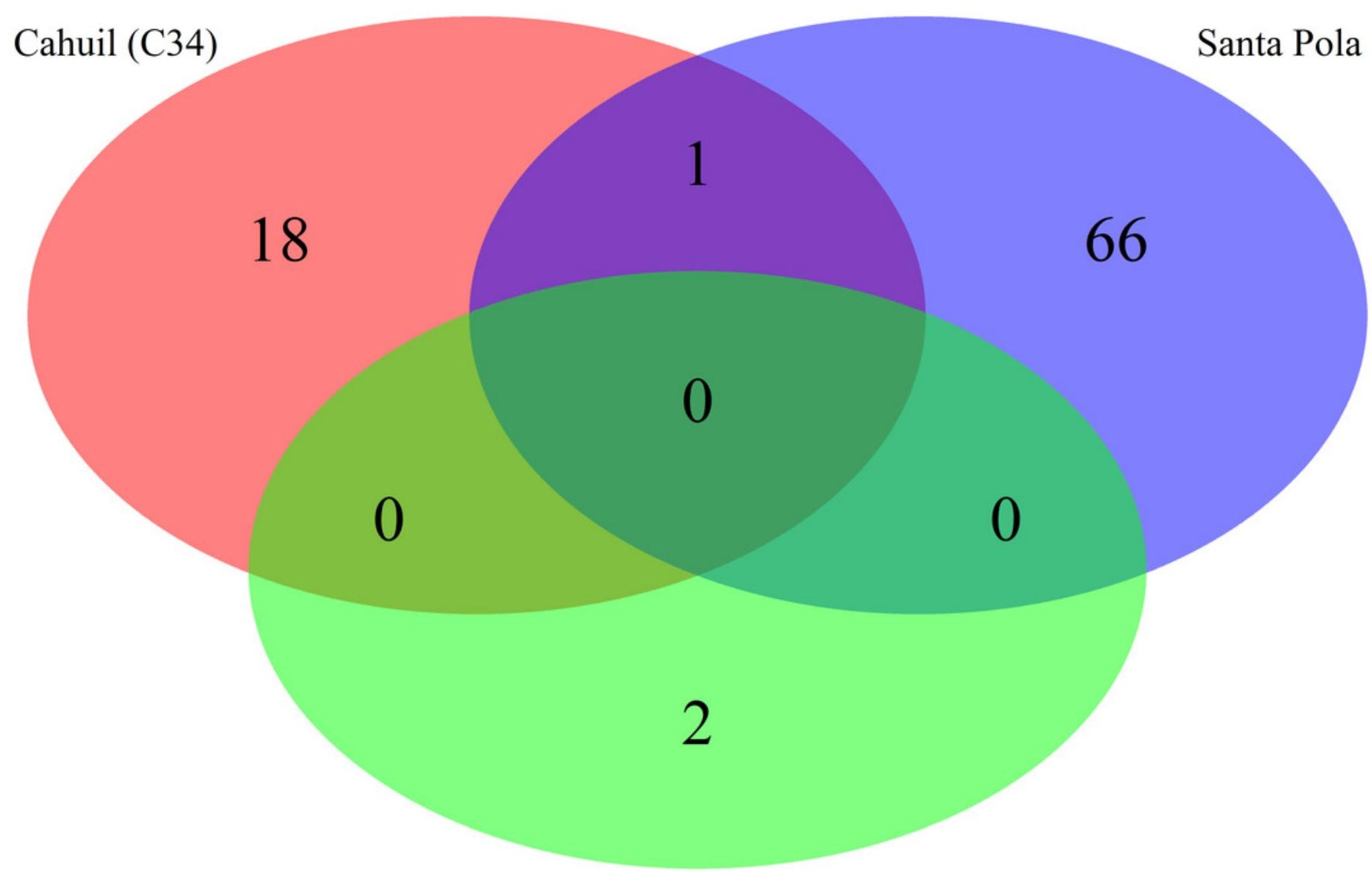

Chula Vista 


\section{Figure 7}

Map of virus-host interactions generated by aligning Crass spacers detected in the combined Santa Pola and Isla Cristina metagenome (Table 3) against a library of haloviral genomes.

The library of haloviral genomes screened is listed in Table S1. Nodes represent either viruses or spacers, while edges represent BLAST alignments linking spacers to viruses. Viruses are marked in blue and spacers in orange. Spacers are listed with both the most likely taxonomic affiliation and sequence ID number (in parentheses). Visualization was performed with Cytoscape.

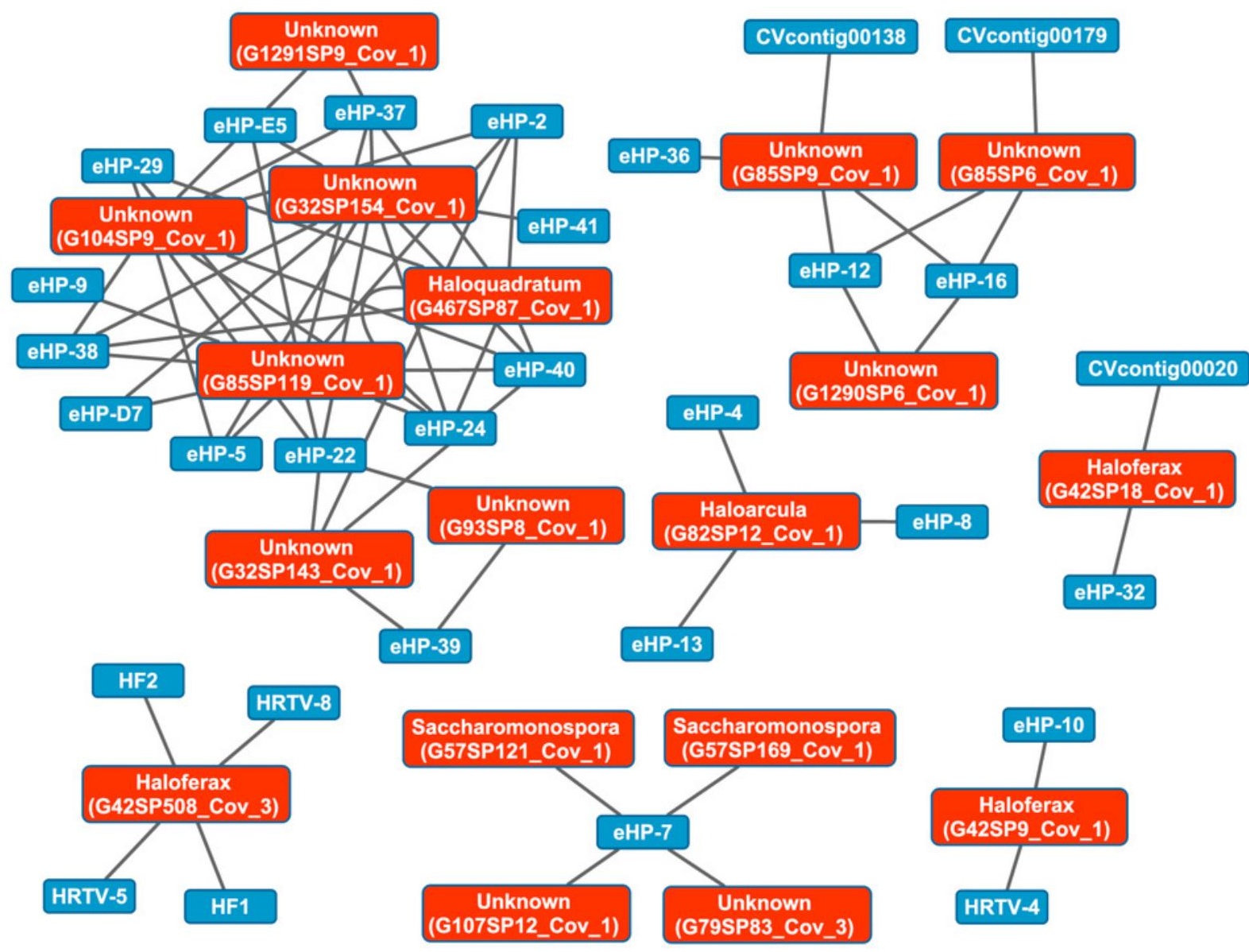




\section{Figure 8 (on next page)}

Map of virus-host interactions generated by aligning spacers detected with referenceguided methods in SS13 (A), SS19 (B), SS33 (C), SS37 (D), IC21 (E), and Cahuil/C34 (F).

The library of haloviral genomes screened is listed in Table S1. Nodes represent either viruses or spacers, while edges represent BLAST alignments linking spacers to viruses. Viruses are marked in blue and spacers in orange. Spacers are listed with both the most likely taxonomic affiliation and sequence ID number (in parentheses). Visualization was performed with Cytoscape. 
A

Pee Hzloquadratum (P1S2)

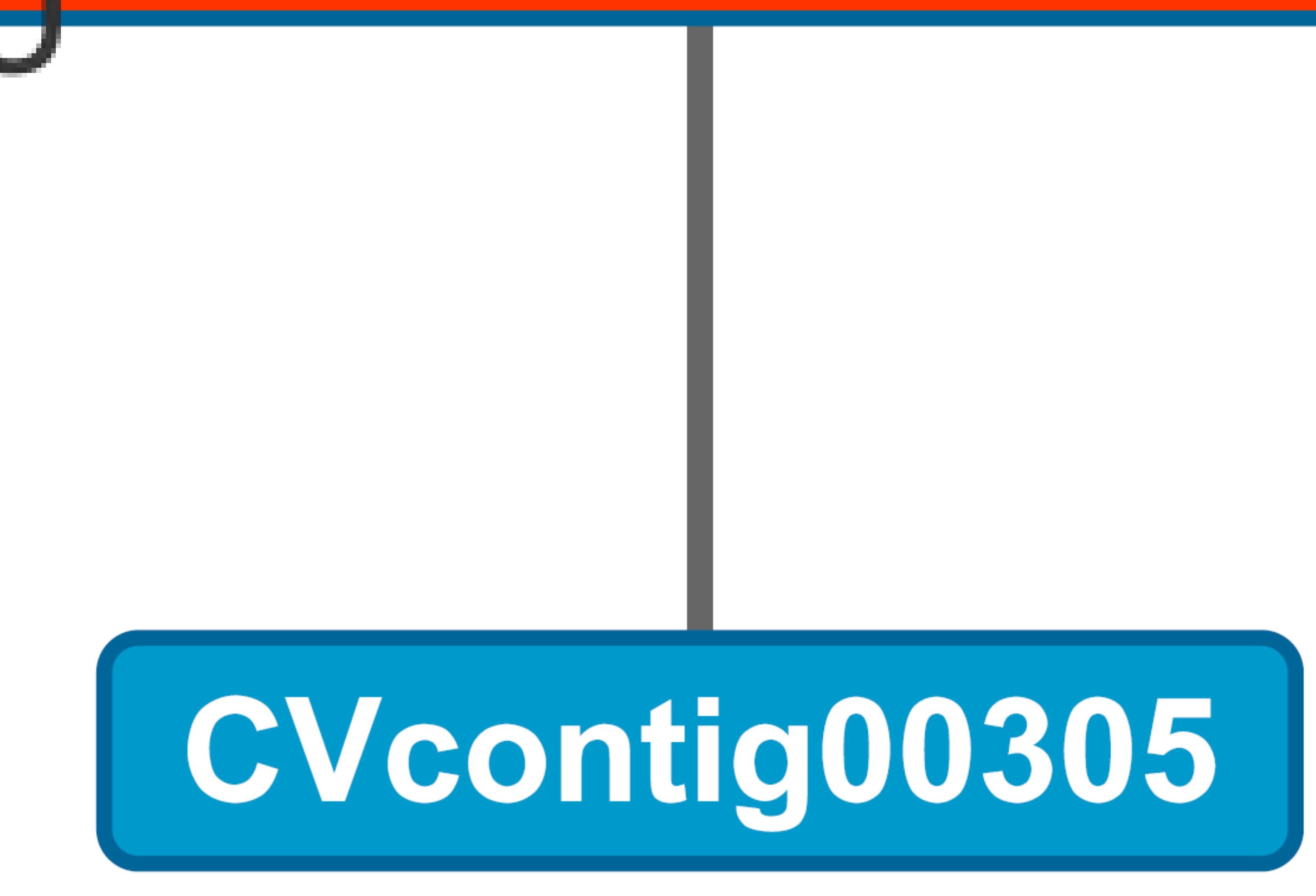

C

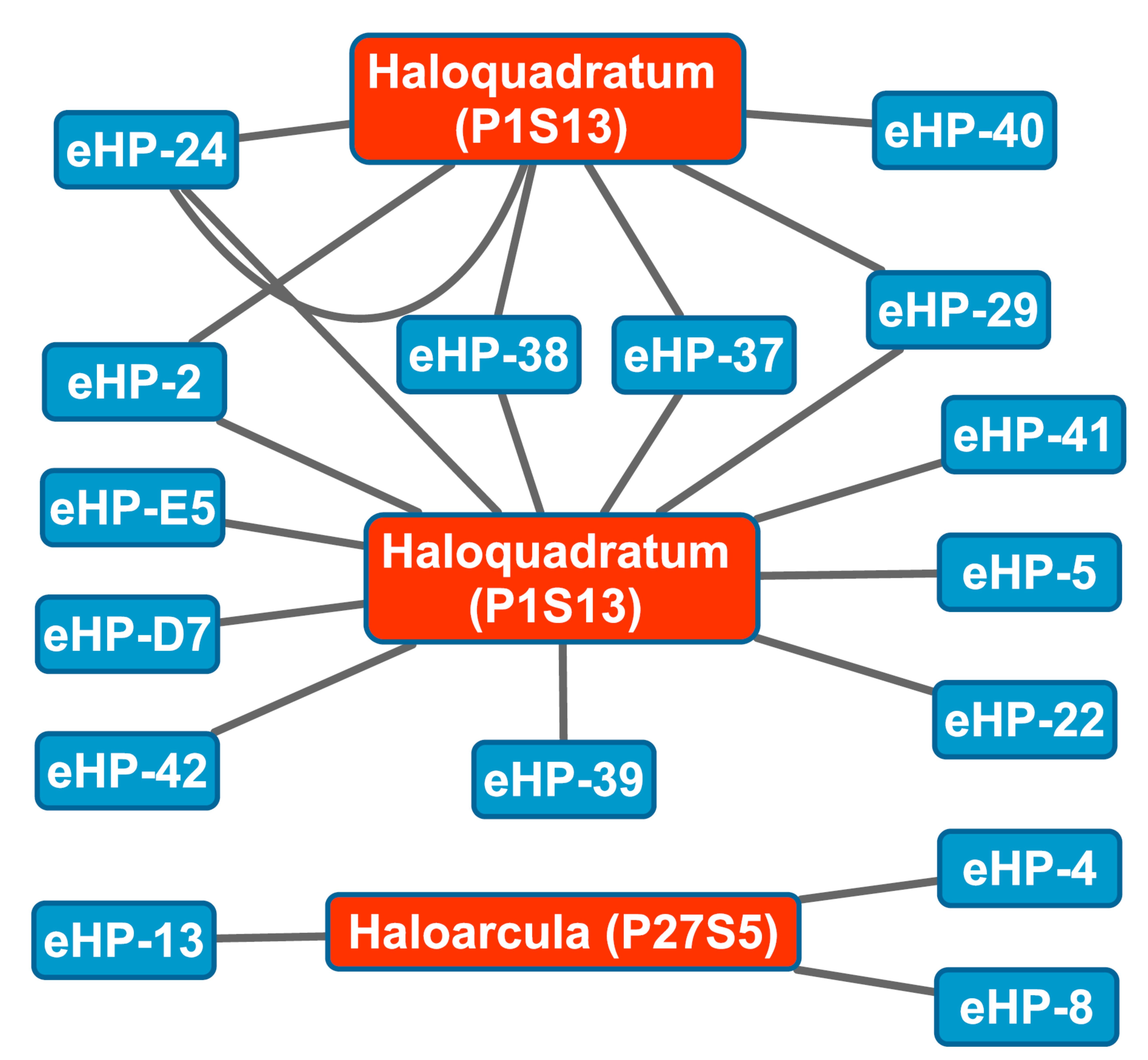

B

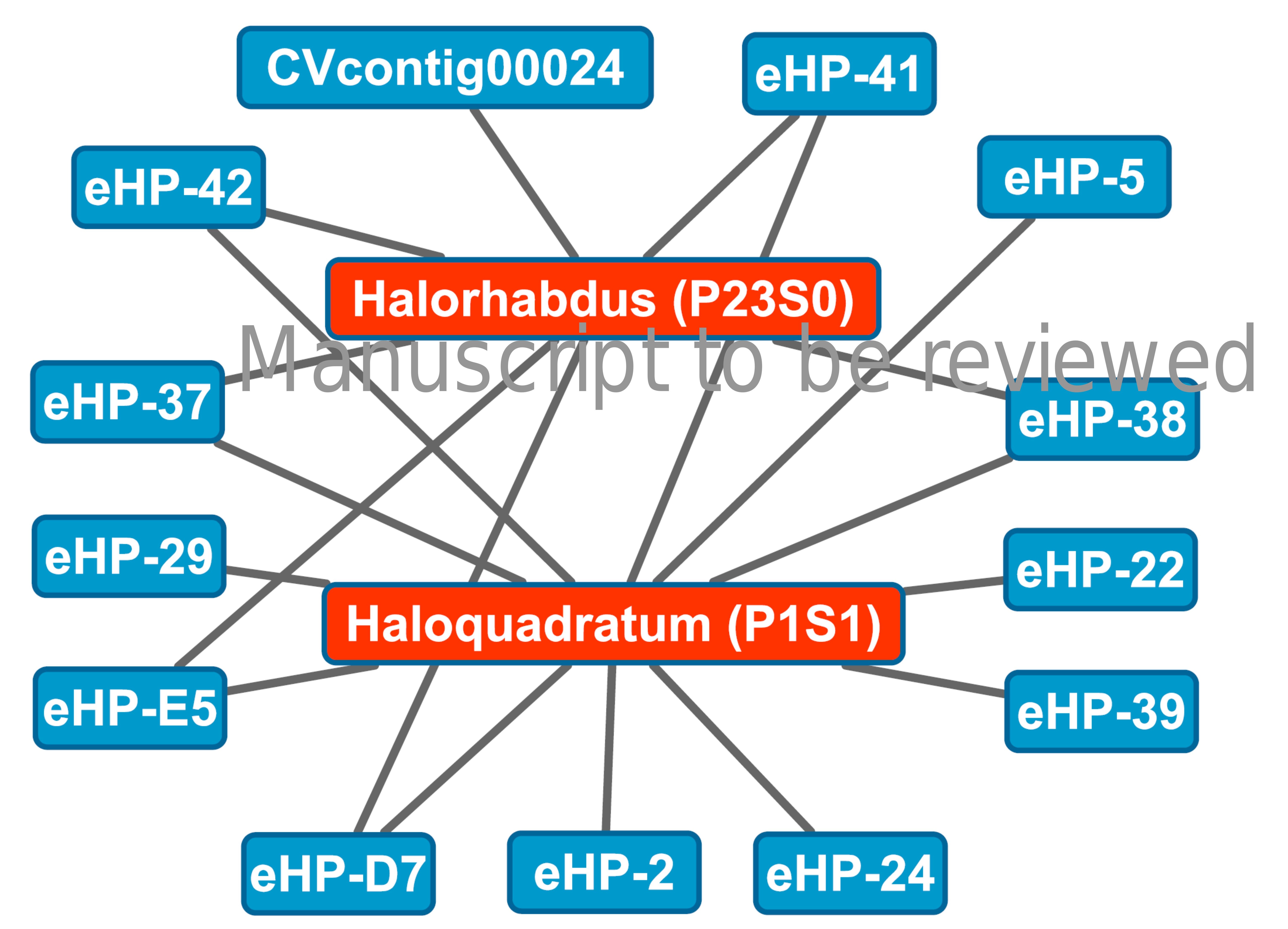

D

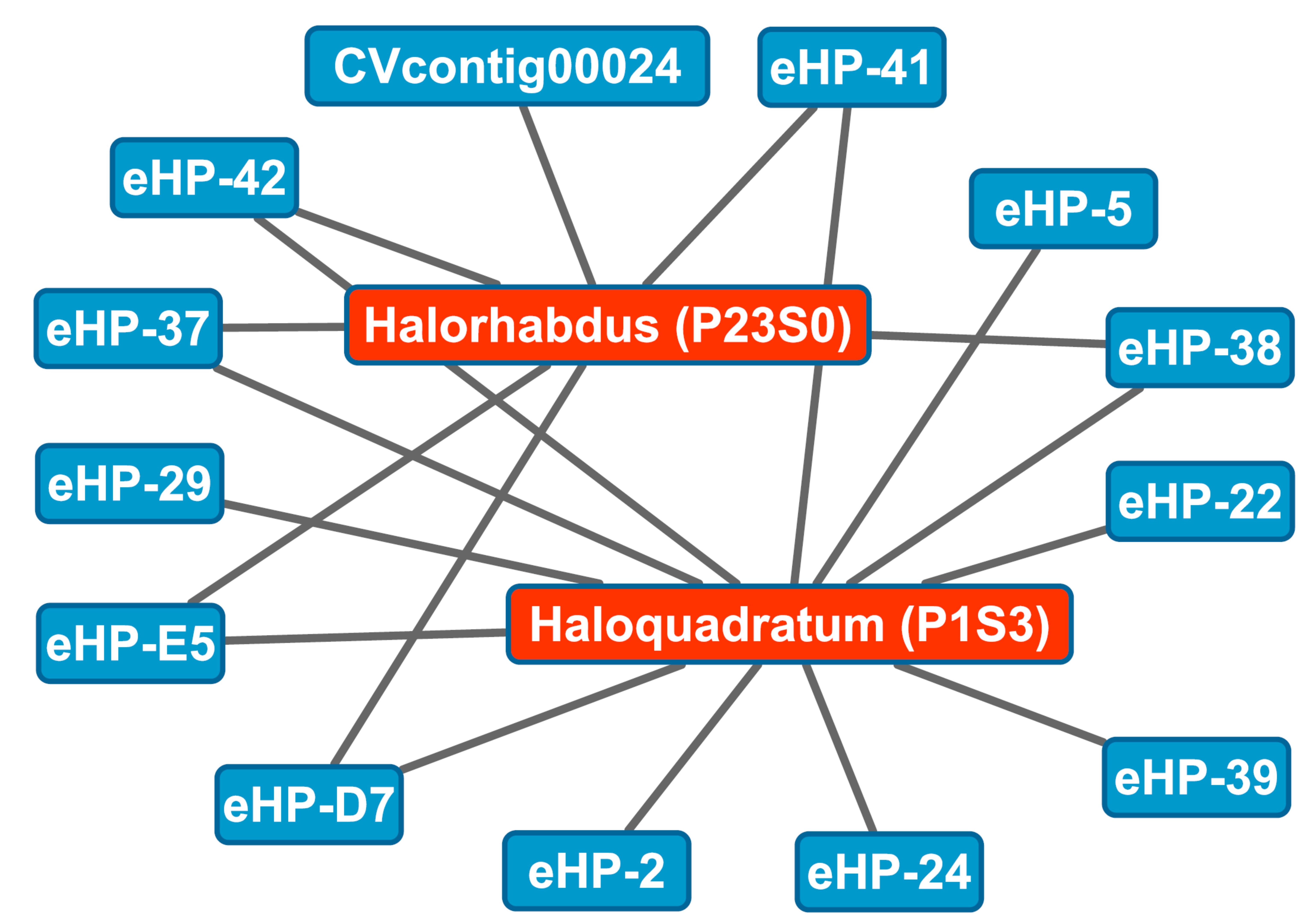

E

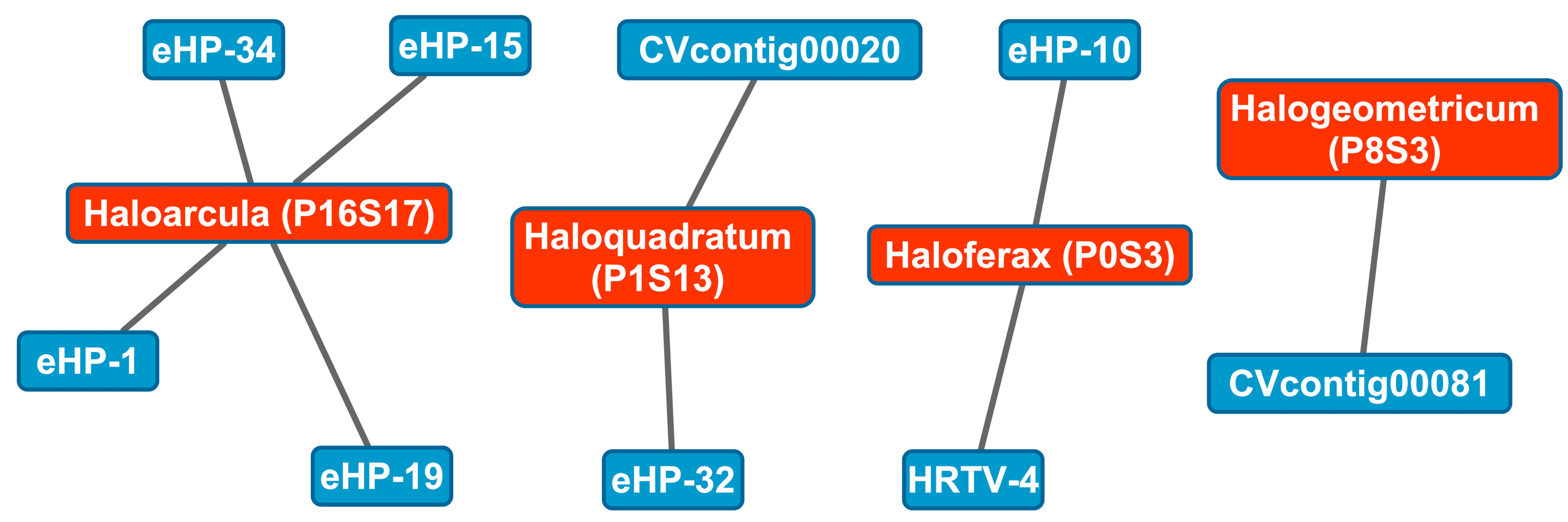

$\mathbf{F}$

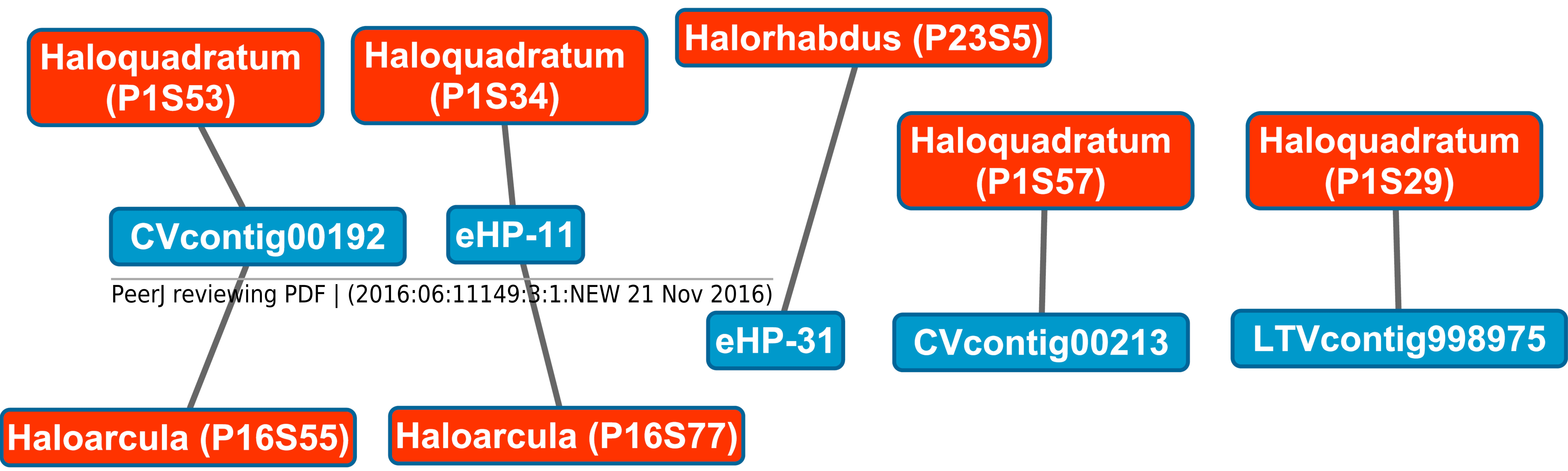


Figure 9 (on next page)

Differences in dimer and trimer usages between host and virus genomes matched with CRISPR spacers.

The difference between each possible dimer and trimer usage was subtracted, squared, and summed across all possible dimers or trimers to generate a measure of dimer or trimer difference. The average dimer usage and trimer usage differences were calculated for all combinations of viruses with a particular host ( $A$ and $B$, respectively) and all virus-host combinations detected for a particular metagenome ( $C$ and $D$, respectively). All averages are reported with a $95 \%$ confidence interval (within two standard error) in black. 


\section{PeerJ}

A

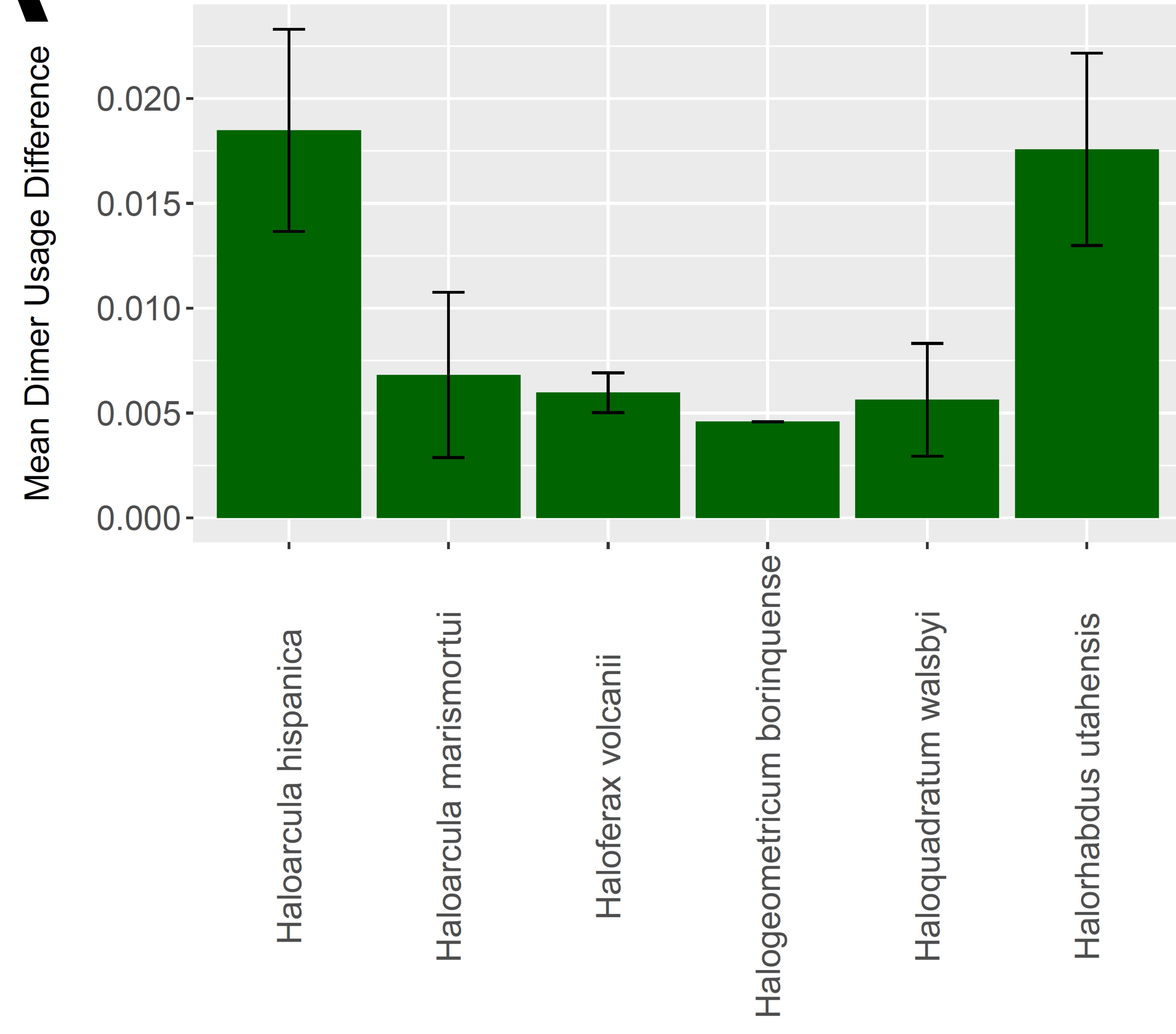

Host
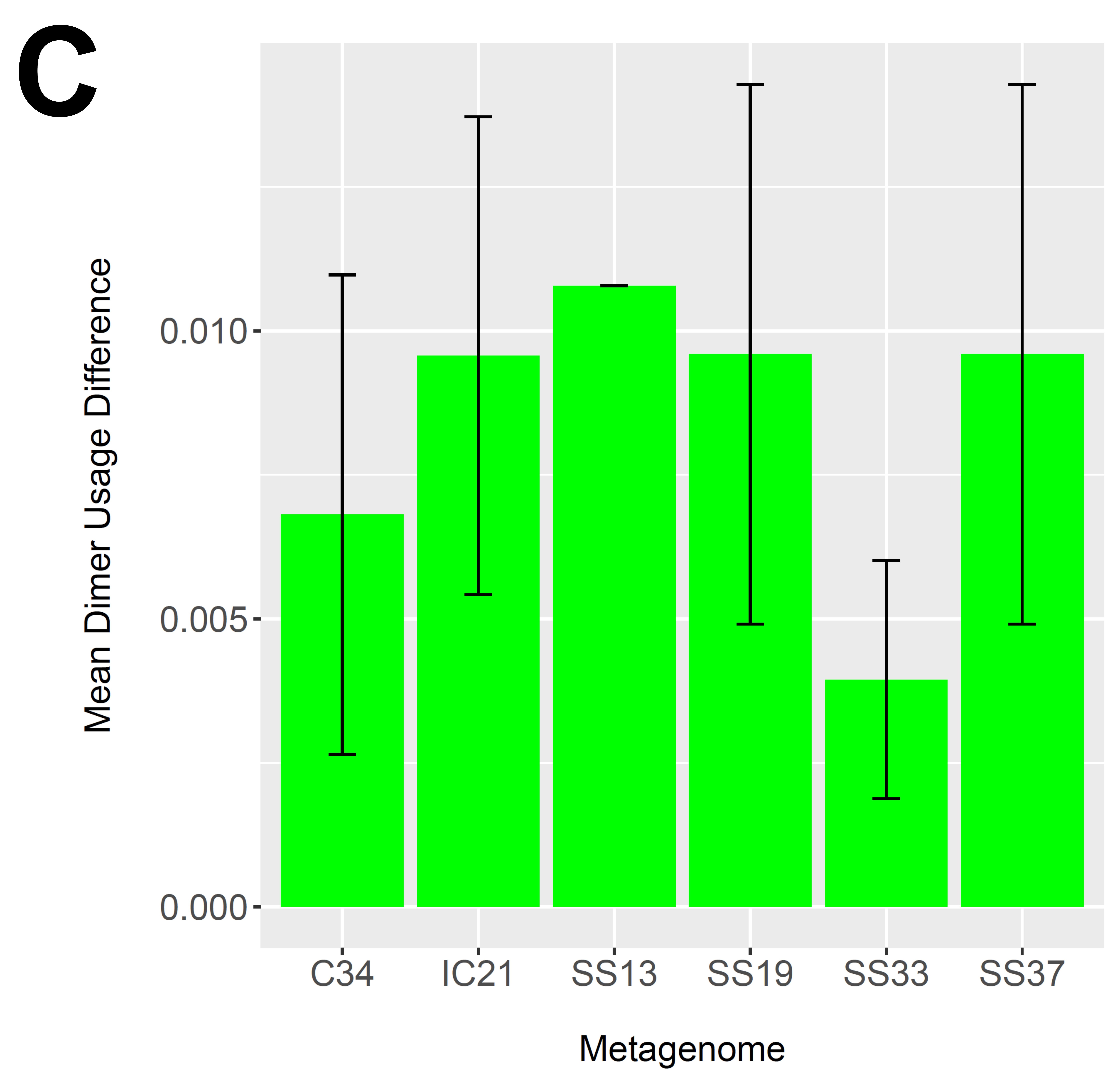

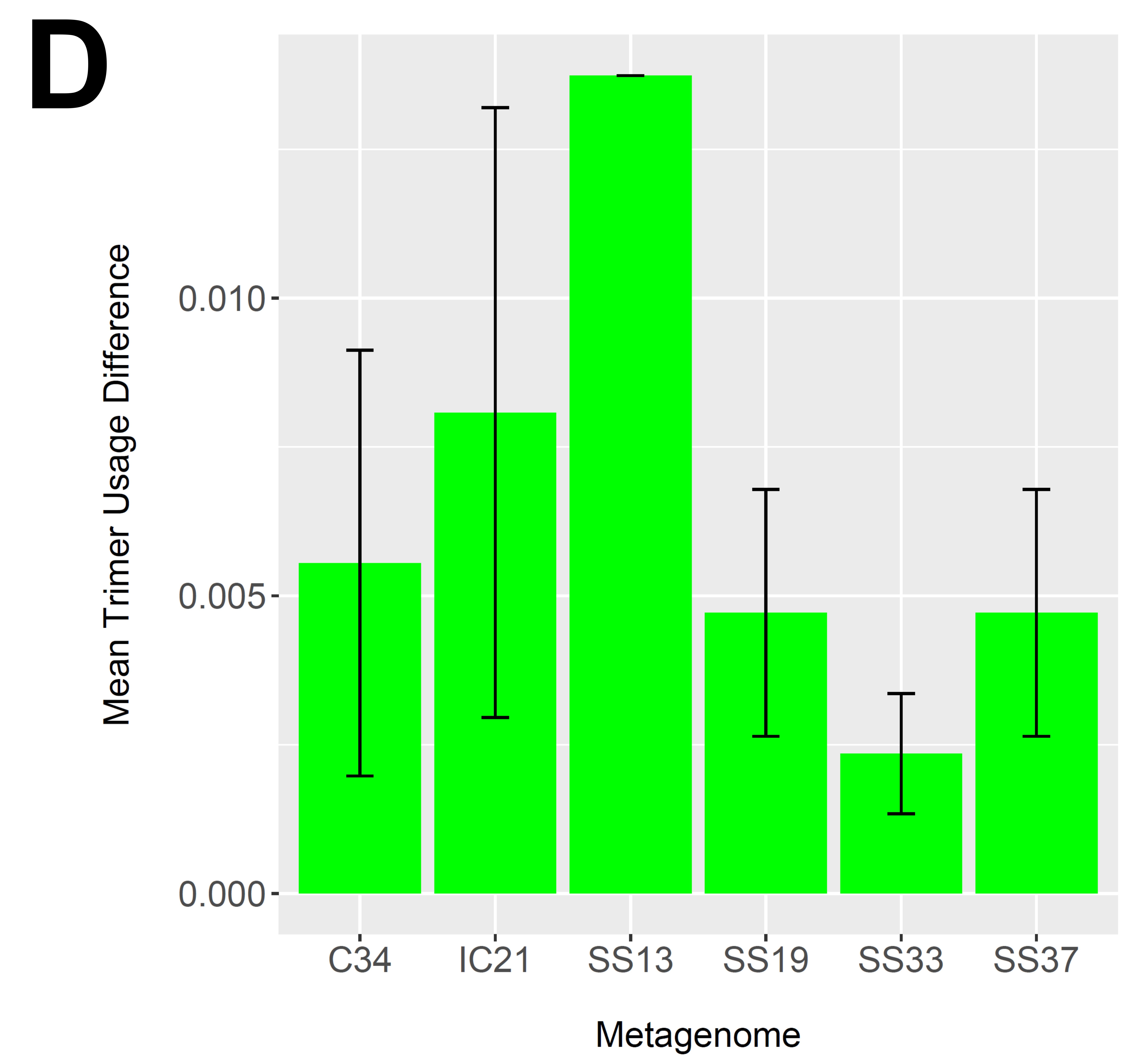


Figure 10 (on next page)

Detection of cas operons in metagenomes and affiliation of detected genes to representative taxa.

Three metagenomes (Cahuil/C34, combined Chula Vista, and combined Santa Pola and Isla Cristina) were assembled and searched for contigs matching cas genes. Detection results are reported for five halobacterial cas operons (A). cas genes detected in no metagenomes are shown in red; genes detected in one metagenome are shown in blue; genes detected in two metagenomes are shown in green (A). Detected contigs assembled with Newbler and Velvet were further aligned against a library of cas genes from three halobacterial orders (Halobacteriales, Haloferacales, and Natrialbales). The taxonomic affiliations of the best hits for each contig were tabulated into profiles for each order and assembly method (B). 
Haloquadratum walsbyi C23 (type I-D)

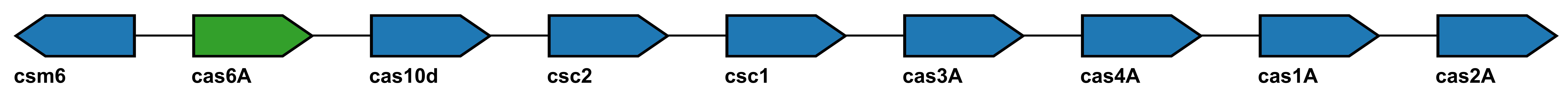

Haloquadratum walsbyi C23 (type I-B)

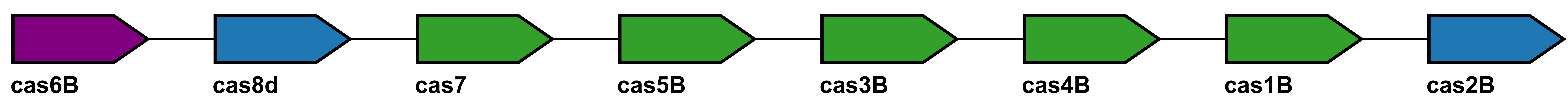

Haloferax volcanii DS2 (type I-B)

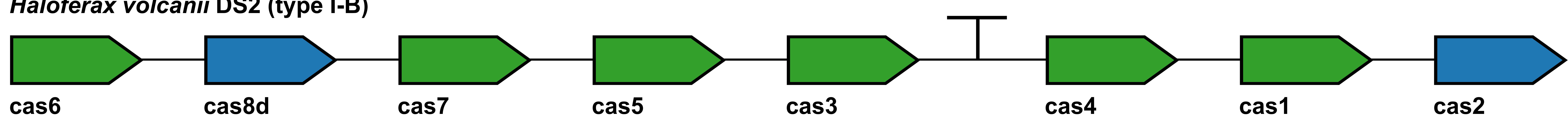

Halorubrum lacusprofundi ATCC 49239 (type I-B)

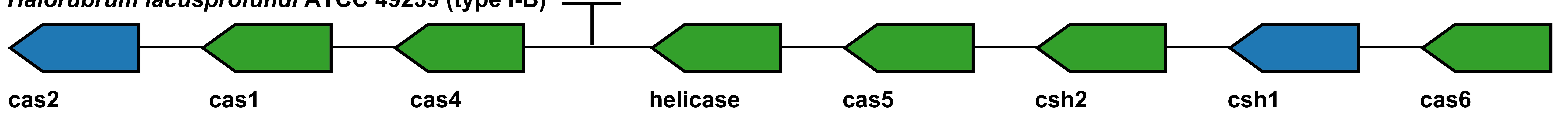

Halorubrum lacusprofundi ATCC 49239 (type I-D)
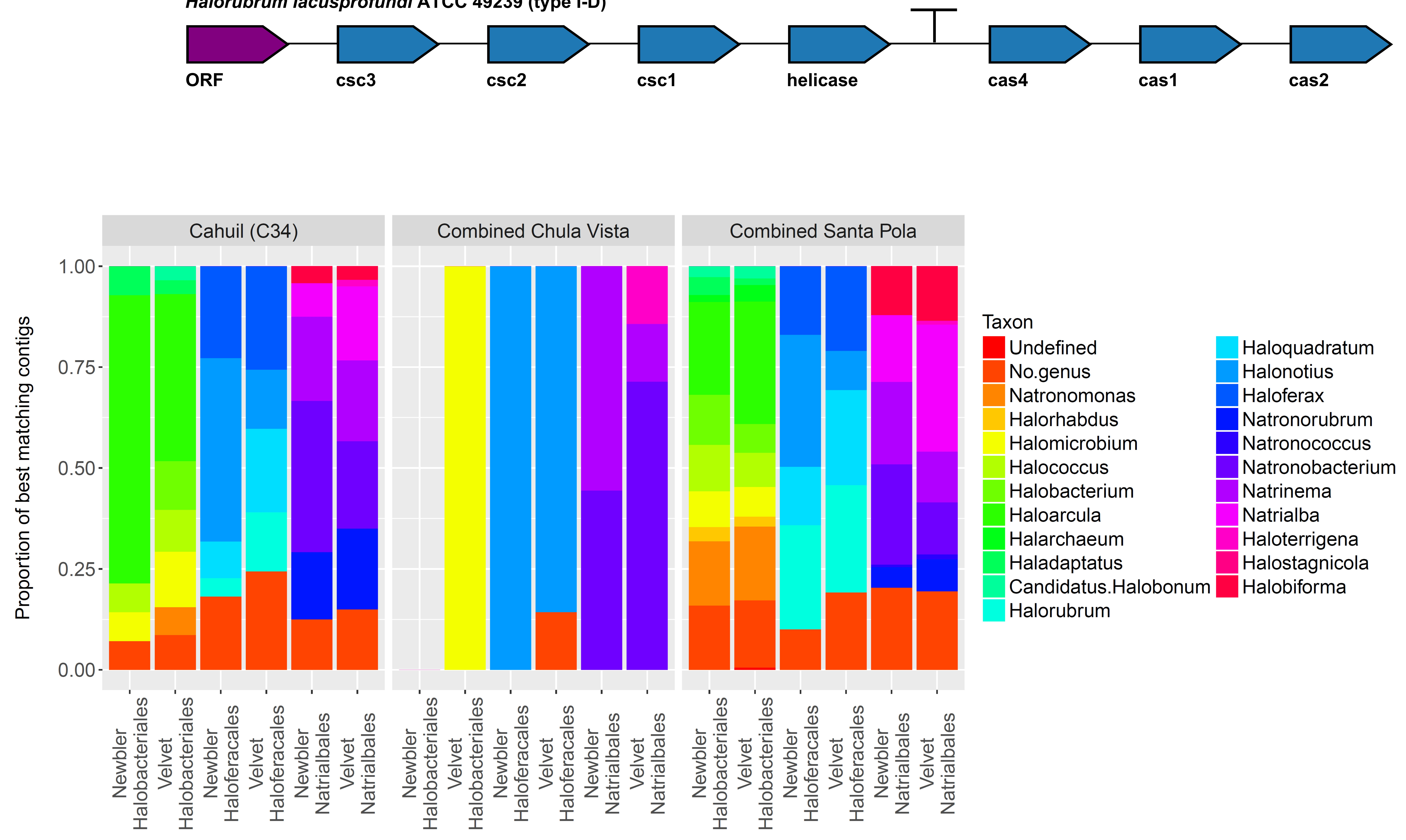

Metagenome 


\section{Table $\mathbf{1}$ (on next page)}

Additional technical information about the Santa Pola, Isla Cristina, and Cahuil solar saltern metagenomes.

This information is provided in the NCBI Sequence Read Archive (SRA) and associated genome announcements (Fernandez et al., 2013; Fernández et al., 2014; Plominsky et al., 2014). All metagenomes were sequenced using 454 technology. Site names (as listed in the Figures) are listed in bold. NCBI SRA accession numbers (SRX for experiment and SRR for run) are included in the table. Combinations of these metagenomes used for further analyses are listed in Table 3. 
1 Table 1: Additional technical information about the Santa Pola, Isla Cristina, and Cahuil solar 2 saltern metagenomes provided in the NCBI Sequence Read Archive (SRA) and associated 3 genome announcements (Fernandez et al., 2013; Fernández et al., 2014; Plominsky et al., 2014). 4 All metagenomes were sequenced using 454 technology. Site names are listed in bold. NCBI 5 SRA accession numbers (SRX for experiment and SRR for run) are included in the table.

6 Combinations of these metagenomes used for further analyses are listed in Table 3.

\begin{tabular}{|c|c|c|c|c|c|c|}
\hline Site & Salinity & Reference & SRX number & $\begin{array}{c}\text { Sequencing } \\
\text { technology }\end{array}$ & $\begin{array}{c}\text { Number } \\
\text { of reads } \\
\text { (millions) }\end{array}$ & $\begin{array}{c}\text { Average } \\
\text { read length } \\
\text { (bp) }\end{array}$ \\
\hline SS13 & $13 \%$ & Fernandez et al., 2013 & SRX328504 & 454 & 1.5 & 479 \\
\hline SS19 & $19 \%$ & Ghai et al., 2011 & SRX024859 & 454 & 0.293 & 504 \\
\hline IC21 & $21 \%$ & Fernandez et al., 2014 & SRX352042 & 454 & 1.2 & 508 \\
\hline SS33 & $33 \%$ & Fernandez et al., 2013 & SRX347883 & 454 & 0.971 & 429 \\
\hline SS37 & $37 \%$ & Ghai et al., 2011 & SRX090229 & 454 & 0.741 & 511 \\
\hline Cahuil & $34 \%$ & Plominsky et al., 2014 & SRX680116 & 454 & 0.222 & 523 \\
(C34) & & & & & & \\
\hline
\end{tabular}

7 


\section{Table 2 (on next page)}

Additional information about the Chula Vista saltern microbial metagenomes obtained from the iMicrobe Collaborative and combined into three metagenomes based on salt concentration.

Site names (as listed in the Figures) are in bold. The datasets can be found at this web address: http://data.imicrobe.us/project/view/58. 
1 Table 2: Additional information about the Chula Vista saltern microbial metagenomes obtained

2 from the iMicrobe Collaborative and combined into three metagenomes based on salt

3 concentration. Site names are in bold. The datasets can be found at this web address:

4 http://data.imicrobe.us/project/view/58.

\begin{tabular}{|l|l|l|l|l|l|}
\hline $\begin{array}{l}\text { Salt content (and } \\
\text { label in figures in } \\
\text { bold) }\end{array}$ & $\begin{array}{l}\text { Sampling dates } \\
\text { corresponding } \\
\text { to included } \\
\text { metagenomes }\end{array}$ & $\begin{array}{l}\text { Sequencing } \\
\text { technology }\end{array}$ & $\begin{array}{l}\text { Number of } \\
\text { reads (millions) }\end{array}$ & $\begin{array}{l}\text { Average read } \\
\text { length (bp) }\end{array}$ & $\begin{array}{l}\text { Total } \\
\text { length } \\
\text { (Mbp) }\end{array}$ \\
\hline $\begin{array}{l}\text { Low (6-8\%) } \\
\text { (CV6-8) }\end{array}$ & $\begin{array}{l}07 / 2004 \\
11 / 10 / 2005 \\
11 / 28 / 2005\end{array}$ & 454 & 0.352 & 95 & 33.4 \\
\hline $\begin{array}{l}\text { Medium (12-14\%) } \\
\text { (CV12-14) }\end{array}$ & $\begin{array}{l}11 / 10 / 2005 \\
11 / 11 / 2005 \\
11 / 16 / 2005 \\
11 / 28 / 2005\end{array}$ & 454 & 0.223 & 99 & 22.1 \\
\hline $\begin{array}{l}\text { High (27-30\%) } \\
\text { (CV27-30) }\end{array}$ & $\begin{array}{l}07 / 2004 \\
11 / 28 / 2005\end{array}$ & 454 & 0.618 & 102 & 63.2 \\
\hline
\end{tabular}

5 


\section{Table 3 (on next page)}

Combined metagenomes, the individual metagenomes that compose them, and how they were used in following analyses.

The Cahuil metagenome (C34) was never included in any combined metagenome used for further analyses. The combined metagenomes were mainly used to improve de novo CRISPR detection, virus-host mapping using these de novo-detected CRISPR spacers, and assembly into contigs for detection of cas operons. These analyses required higher depth metagenomes; for example, no de novo-detected CRISPR spacers in several individual Santa Pola metagenomes mapped to any viruses in the halovirus library. The combined Chula Vista viral metagenome was used to assemble viral contigs included in the haloviral library (Table S1). 
1 Table 3: Combined metagenomes, the individual metagenomes that compose them, and how they 2 were used in following analyses. The Cahuil metagenome (C34) was never included in any 3 combined metagenome used for further analyses. The combined metagenomes were mainly used 4 to improve de novo CRISPR detection, virus-host mapping using these de novo-detected 5 CRISPR spacers, and assembly into contigs for detection of cas operons. These analyses 6 required higher depth metagenomes; for example, no de novo-detected CRISPR spacers in 7 several individual Santa Pola metagenomes mapped to any viruses in the halovirus library. The 8 combined Chula Vista viral metagenome was used to assemble viral contigs included in the 9 haloviral library (Table S1).

\begin{tabular}{|c|c|c|}
\hline Combined metagenome & Individual metagenomes included & How they were analyzed \\
\hline $\begin{array}{l}\text { Santa Pola and Isla Cristina } \\
\text { (Combined SP) }\end{array}$ & $\begin{array}{l}\text { SS13, SS19, IC21, SS33, and } \\
\text { SS37 }\end{array}$ & $\begin{array}{l}\text { - De novo CRISPR detection } \\
\text { with Crass (Figure 4) } \\
\text { - Reference-guided CRISPR } \\
\text { detection (Figure 4) } \\
\text { - Comparing DRs detected with } \\
\text { the de novo CRISPR detection } \\
\text { method Crass (Figure 6) } \\
\text { - Virus-host mapping using de } \\
\text { novo-detected CRISPR spacers } \\
\text { (Figure 7) } \\
\text { - Assembly to detect cas operons } \\
\text { (Figure 10) }\end{array}$ \\
\hline $\begin{array}{l}\text { Chula Vista } \\
\text { (Combined CV) }\end{array}$ & CV6-8, CV12-14, and CV27-30 & $\begin{array}{l}\text { - De novo CRISPR detection } \\
\text { with Crass (Figure 4) } \\
\text { - Reference-guided CRISPR } \\
\text { detection (Figure 4) } \\
\text { - Comparing DRs detected with } \\
\text { the de novo CRISPR detection } \\
\text { method Crass (Figure 6) } \\
\text { - Assembly to detect cas operons } \\
\text { (Figure 10) }\end{array}$ \\
\hline $\begin{array}{l}\text { Chula Vista combined viral } \\
\text { metagenome }\end{array}$ & Chula Vista viral metagenomes & $\begin{array}{l}\text { - Assembly of Chula Vista viral } \\
\text { contigs included in haloviral } \\
\text { library (Figures } 7 \text { and 8; Table } \\
\text { S1) }\end{array}$ \\
\hline
\end{tabular}

